\title{
Band gap property analysis of periodic plate structures under general boundary conditions
}

\section{using spectral-dynamic stiffness method}

Guoyong Jin ${ }^{*}$, Chunyu Zhang ${ }^{*}$, Tiangui Ye, Jialiang Zhou

\begin{abstract}
Vibration band gap properties of periodic rectangular plate structures with general boundary conditions are studied using the spectral-dynamic stiffness method (S-DSM). Material and dimensional properties are assumed to vary periodically in the whole plate structure. In the S-DSM, the general solution governing each unit plate is firstly derived with the aid of spectral method and then the dynamic stiffness matrix is established by substituting the general solution into corresponding boundary equations. Finally, the global spectral dynamic matrix for the whole periodic plate structure is assembled in a similar way to the finite element method (FEM). The harmonic responses are calculated to illustrate the band gap properties of the periodic structures. By comparing the results obtained by the FEM, it can be verified that the S-DSM is of superior accuracy and convergence rate. A parametric study is also conducted to investigate the effects of the material properties, geometrical parameters and structural damping on the vibration band gap behaviors of the periodic structures.
\end{abstract}

Keywords: Spectral dynamic stiffness method; Vibration band gap; Periodic plate structure; General boundary conditions 


\section{Introduction}

The propagation of elastic waves in periodic systems has received much attention over the years [1-8]. More recently, there has been an active area of research in a special type of inhomogeneous materials and geometries (the so-called phononic crystals) whose elastic coefficients or geometrical parameters vary periodically in space. Many remarkable mechanic properties of such structures have been discovered and investigated. Among them, the band gap property in frequency domain is one of the most distinctive properties which is caused by the elastic waves reflected back and forth between the interfaces of the adjacent cells when they propagate in the periodic structures. In the band gap frequency range, nearly no elastic wave can propagate through the periodic structure. The vibration band gap properties enable the periodic structure to be widely used in engineering fields, such as, frequency filters, noise controls, vibrationless environments for high-precision mechanical systems, etc. [4, 13, 14].

A variety of both theoretical and numerical studies have been conducted to investigate the wave filtering behavior in periodic structures. For example, Yan and Wang $[9,10,12]$ developed a wavelet-based method (WBM) to study the elastic band gaps of phononic crystals. The eigen-equation for the phononic crystals was derived by expanding the displacement fields in the wavelet basis functions. Based on the transfer matrix method (TMM), Ruzzene and Marino [15, 16] studied the dynamic behaviors of the periodic rods with shape memory inserts which can act as the filters to weaken the elastic wave propagation along the rods. Yu et al. [17] extended the TMM to calculate the band gaps of flexural waves in the Euler-Bernoulli beam and studied its transverse vibration band gap behaviors. To investigate the flexural vibration band gap properties of the periodic beams, Xiang and Shi [18] derived the theoretical equations of beam by employing the Bloch-Floquet theorem and then solved the equations using the differential quadrature method. 
Olhoff et al. [19] analyzed a repeated inner segment of the optimized beams using the waveguide

finite element method (WFEM) to study the band-gap for travelling waves. The authors aimed to maximize the band gaps between two adjacent natural frequencies and to study the associated creation of periodicity of the optimized beam designs. Hussein et al. [20] designed the target frequency band structures characterizing longitudinal wave motion of one-dimensional periodic unit cell by using a multi-objective genetic algorithm. The design approach provided practical guides for forming bounded structures with advanced vibration, shock isolation and filtering characteristics. Considerable vibration studies on varies of periodic cylindrical shell structures are also conducted in recent years [21-23]. Shen et al. [24] and Wen et al. [25] took the advantages of band gap properties of periodic composite pipes to provide a novel avenue for vibration control and noise reduction in complex piping systems. Sorokin and Ershova [26] employed the Floquet theory and boundary integral equations to study the steady-state free vibration of non-uniform elastic cylindrical shells with and without internal heavy fluid loading. Shen et al. [27, 28] illustrated the mechanism of wave propagation and vibration transmission in functionally graded material periodic cylindrical shell and studied its capability to control sound and vibration.

The structures of periodically placed plates are commonly encountered in various practical engineering. Much effort has been devoted to investigating the dynamic characteristic of such periodic plate structures in the past decades. In the early years, Sigalas and Economou [29] calculated the elastic waves propagation in plates with solid inclusions placed periodically in the host plate. Langley and Smith [30] used the statistical energy analysis (SEA) to study the effects of disorder in the stiffener spacing on the high frequency vibration transmission through a periodic plate system. In recent years, Wang et al. [31] developed an improved FEM to study the physical mechanisms for the formation of periodiclly stiffened plate structures with any number or 
orientation of stiffeners. Wu et al. [32, 33] applied the spectral element method (SEM) to investigate

the dynamic behavior of periodic plate structures with classic plate theory (CPT) and Mindlin plate theory (MPT). But both the improved FEM and SEM have their limitations. For the FEM, the number of element should be large enough to obtain credible results in high-frequency range. In addition, computational efficiency and memory space will be unacceptable if the element number becomes very large. Although the SEM is of excellent accuracy and efficiency, it is only suitable for limited boundary conditions for plate structures, i.e., at least two opposite simply supported boundaries. Recently, the dynamic stiffness method (DSM) has drawn more and more attention due to its high computational efficiency and accuracy compared with the FEM resulting from the exact shape functions and lower degrees of freedom. And it has been developed for a bunch of one-dimensional (1D) elements such as rods and beams [34-36] and two dimensional (2D) rectangular plates using various plate theories $[37,38]$. However, for plate structures, the traditional DSM is only available for the cases where at least a pair of opposite boundaries are simply supported, i.e. Levy plates. More recently, Liu and Banerjee [39] developed a novel S-DSM by introducing the idea of general spectral method, which had removed the boundary constraints of the traditional DSM. This idea certainly opened up a new way to solve vibration problems of plate structures in an accurate and efficient manner. Afterwards, Banerjee and his co-workers extended and extended the S-DSM to study the free vibration of composite plate structures and plate assemblies with arbitrary non-uniform elastic supports mass attachments and elastic coupling constraints [40-42].

In the present work, the S-DSM is employed to calculate the forced vibration responses of periodic plate structures under general boundary conditions and investigate the band gap behaviors in frequency domain. The S-DSM is more efficient than the FEM and can cope with more complex 
boundary conditions than the SEM. This paper is arranged as follow: in Section 2, the formulation

of the dynamic stiffness matrix of the periodic plate structure is introduced briefly, after which the applications of external load and arbitrary prescribed boundary conditions are given. In Section 3 , firstly, several numerical examples are given to validate the accuracy and convergence of the current results. Then, a parametric study is conducted to investigate the band gap behaviors of the periodic palate structures under the effects of material and dimensional properties. Finally, the conclusions of this paper are given in Section 4.

\section{Mathematical formulation}

The periodic plate structure shown in Fig. 1 is considered in this work. The system consists of $N$ repetitions of alternating plates $A$ and $B$. Plates $A$ and $B$ are made of different material properties or geometric dimensions. A set of parallel edges are under general boundary conditions and the other set of edges are free or coupled with the edges of the adjacent plates.

\subsection{Governing differential equation}

Fig.2 shows a rectangular Kirchhoff plate in its local coordinate system. The length, width and thickness of the plate element are $2 a, 2 b$ and $h$, respectively. In this study, the plate is assumed to be homogeneous, isotropic, elastic and of uniform thickness. The thickness of the plate is much smaller than the other two dimensions, and the inertia of shear and rotation are supposed to be negligible. Then, based on the Kirchhoff thin plate theory, the differential equation can be described as [43]

$$
D\left(\frac{\partial^{4} w}{\partial x^{4}}+2 \frac{\partial^{4} w}{\partial x^{2} \partial y^{2}}+\frac{\partial^{4} w}{\partial y^{4}}\right)+\rho h\left(\frac{\partial^{2} w}{\partial t^{2}}\right)=0
$$

where $D=E h^{3} /\left[12\left(1-v^{2}\right)\right]$ is the bending stiffness, $E$ is the Young's modulus, $v$ and $\rho$ are the Poisson's ratio and density of the plate respectively. 
By introducing the harmonic representation, the transverse displacement can be given as

$$
w(x, y, t)=W(x, y) e^{i \omega t}
$$

where $\omega$ is the angular frequency, $W(x, y)$ is the amplitude of the transverse displacement in frequency domain. By substituting Eq. (2) into Eq. (1), one can get

$$
D\left(\frac{\partial^{4} W}{\partial x^{4}}+2 \frac{\partial^{4} W}{\partial x^{2} \partial y^{2}}+\frac{\partial^{4} W}{\partial y^{4}}\right)-\rho h \omega^{2} W=0
$$

Based on the thin plate assumptions, the rotations of the transverse normal, bending moments and transverse shear forces can be written as

$$
\begin{gathered}
\varphi_{x}=-\frac{\partial W}{\partial x}, \\
\varphi_{y}=-\frac{\partial W}{\partial y}, \\
M_{x}=-D\left(\frac{\partial^{2} W}{\partial x^{2}}+v \frac{\partial^{2} W}{\partial y^{2}}\right), \\
M_{y}=-D\left(\frac{\partial^{2} W}{\partial y^{2}}+v \frac{\partial^{2} W}{\partial x^{2}}\right), \\
V_{y}=-D\left(\frac{\partial^{3} W}{\partial x^{3}}+(2-v) \frac{\partial^{2} W}{\partial x \partial y^{2}}\right), \\
\left.\partial y^{3}+(2-v) \frac{\partial^{2} W}{\partial y \partial x^{2}}\right),
\end{gathered}
$$

\subsection{General solution}

In the S-DSM, the general solution of Eq. (3) can be sought according to the method of separation of variables [39]

$$
W(x, y)=X(x) Y(y)=C e^{q x+p y}
$$

Where $C, q$ and $p$ are arbitrary coefficient and wave numbers along $x$ and $y$ directions respectively. By submitting Eq. (11) into Eq. (3), the general solution $W(x, y)$ of the governing differential 
equation can be obtained based on Euler's formula. For further consideration of the symmetry/antisymmetry properties of the plate structure, the general expression of $W(x, y)$ can be split into four contributions: symmetric-symmetric (00), symmetric - antisymmetric (01), antisymmetric - symmetric (10), antisymmetric - antisymmetric (11) as

$$
W(x, y)=\sum_{k, j \in[0,1\}} W^{k j}(x, y)=W^{00}+W^{01}+W^{10}+W^{11}
$$

where all the four parts of the solution take the unified concise form:

$$
\begin{aligned}
W^{k j}(x, y)= & \sum_{m \in M}\left[A_{1 k m} \mathcal{H}_{j}\left(p_{1 k m} y\right)+A_{2 k m} \mathcal{H}_{j}\left(p_{2 k m} y\right)\right] \Xi_{k}\left(\alpha_{k m} x\right) \\
& +\sum_{n \in N}\left[B_{1 j n} \mathcal{H}_{k}\left(q_{1 j n} x\right)+B_{2 j n} \mathcal{H}_{k}\left(q_{2 j n} x\right)\right] \Xi_{j}\left(\beta_{j n} y\right)
\end{aligned}
$$

\subsection{Dynamic stiffness matrix}

\subsubsection{Dynamic stiffness matrix for individual plate structure}

The detailed formulation of the dynamic stiffness matrix for the individual plate structure has been detailedly deduced by Liu and Banerjee [39]. In this section, only the main strategy of the derivation procedure is introduced.

As given in Eq. (12), the general solution is spilt into four parts with different symmetry/antisymmetry properties, and likewise, the arbitrarily prescribed boundary conditions are also decomposed into four $k j$ parts defined in $\Omega_{1}$ (first quarter of $\Omega$, see Fig.3 (a)) as shown in Fig. 3(b). Then, the boundary conditions are expressed using the modified Fourier series as

$$
\left[\begin{array}{c}
W_{a}^{k j} \\
W_{b}^{k j} \\
\phi_{a}^{k j} \\
\phi_{b}^{k j}
\end{array}\right]=\left[\begin{array}{c}
\sum_{n \in N} W_{a j n}^{k j} \frac{\Xi_{j}\left(\beta_{j n} y\right)}{\sqrt{\zeta_{j n} b}} \\
\sum_{m \in M} W_{b k m}^{k j} \frac{\Xi_{k}\left(\alpha_{k m} x\right)}{\sqrt{\zeta_{k m} a}} \\
\sum_{n \in N} \phi_{a j n}^{k j} \frac{\Xi_{j}\left(\beta_{j n} y\right)}{\sqrt{\zeta_{j n} b}} \\
\sum_{m \in M} \phi_{b k m}^{k j} \frac{\Xi_{k}\left(\alpha_{k m} x\right)}{\sqrt{\zeta_{k m}} a}
\end{array}\right], \quad\left[\begin{array}{c}
V_{a}^{k j} \\
V_{b}^{k j} \\
M_{a}^{k j} \\
M_{b}^{k j}
\end{array}\right]=D\left[\begin{array}{c}
\sum_{n \in N} V_{a j n}^{k j} \frac{\Xi_{j}\left(\beta_{j n} y\right)}{\sqrt{\zeta_{j n} b}} \\
\sum_{m \in M} V_{b k m}^{k j} \frac{\Xi_{k}\left(\alpha_{k m} x\right)}{\sqrt{\zeta_{k m} a}} \\
\sum_{n \in N} M_{a j n}^{k j} \frac{\Xi_{j}\left(\beta_{j n} y\right)}{\sqrt{\zeta_{j n} b}} \\
\sum_{m \in M} M_{b k m}^{k j} \frac{\Xi_{k}\left(\alpha_{k m} x\right)}{\sqrt{\zeta_{k m} a}}
\end{array}\right]
$$

where $W_{a j n}^{k j}, W_{b k m}^{k j}, \phi_{a j n}^{k j}, \phi_{a j n}^{k j}, V_{a j n}^{k j}, V_{b k m}^{k j}, M_{a j n}^{k j}$ and $M_{b k m}^{k j}$ are the Fourier coefficients. 
On the basis of the relationships between the displacement and force given in Eq. (4-10), the

unknown coefficients $\tilde{A}_{1 k m}, \tilde{A}_{2 k m}, \tilde{B}_{1 j n}$ and $\tilde{B}_{2 j n}$ in the general solution can be determined by substituting Eq. (13) into Eq. (14). Subsequently, an infinite sets of algebraic equations can be obtained by substituting the determined coefficients into Eq. (14), which can be summarized into matrix form as

$$
\boldsymbol{f}^{k j}=\boldsymbol{K}^{k j} \boldsymbol{d}^{k j}
$$

In Eq. (15), the Fourier coefficient vectors $\boldsymbol{f}^{k j}$ and $\boldsymbol{d}^{k j}$ are of the boundary conditions on $B_{a}$ and $B_{a}$ of the quarter plate as shown in Fig. 3(b). Similarly, the corresponding Fourier coefficient vectors of the force and displacement on the boundary lines of the entire plate can be given as

$$
\boldsymbol{d}=\left[\boldsymbol{d}_{1}, \boldsymbol{d}_{2}, \boldsymbol{d}_{3}, \boldsymbol{d}_{4}\right]^{T}, \quad \boldsymbol{f}=\left[\boldsymbol{f}_{1}, \boldsymbol{f}_{2}, \boldsymbol{f}_{3}, \boldsymbol{f}_{4}\right]^{T}
$$

where

$$
\boldsymbol{d}_{i}=\left[\boldsymbol{W}_{i}^{0}, \boldsymbol{W}_{i}^{1}, \Phi_{i}^{0}, \Phi_{i}^{1}\right]^{T}, \boldsymbol{f}_{i}=\left[\boldsymbol{V}_{i}^{0}, \boldsymbol{V}_{i}^{1}, \boldsymbol{M}_{i}^{0}, \boldsymbol{M}_{i}^{1}\right]^{T}
$$

Then, by introduction of the transfer matrix $\boldsymbol{T}$ [39], one can establish the relationships between $\boldsymbol{f}, \boldsymbol{d}$ and $\boldsymbol{f}^{k j}, \boldsymbol{d}^{k j}$ as

$$
\boldsymbol{d}=\boldsymbol{T}\left[\boldsymbol{d}^{00}, \boldsymbol{d}^{01}, \boldsymbol{d}^{10}, \boldsymbol{d}^{11}\right]^{T}, \boldsymbol{f}=\boldsymbol{T}\left[\boldsymbol{f}^{00}, \boldsymbol{f}^{01}, \boldsymbol{f}^{10}, \boldsymbol{f}^{11}\right]^{T}
$$

Finally, the dynamic matrix $\boldsymbol{K}$ for the individual plate structure can be obtained by integrating Eq. (15-18), which is reconstructed in the following form

$$
\boldsymbol{f}=\boldsymbol{K} \boldsymbol{d}
$$

where

$$
\boldsymbol{K}=\frac{1}{2} \boldsymbol{T}\left[\begin{array}{cccc}
\boldsymbol{K}^{00} & \boldsymbol{O} & \boldsymbol{O} & \boldsymbol{O} \\
\boldsymbol{O} & \boldsymbol{K}^{01} & \boldsymbol{O} & \boldsymbol{O} \\
\boldsymbol{O} & \boldsymbol{O} & \boldsymbol{K}^{10} & \boldsymbol{O} \\
\boldsymbol{O} & \boldsymbol{O} & \boldsymbol{O} & \boldsymbol{K}^{11}
\end{array}\right] \boldsymbol{T}^{T}
$$




\subsubsection{Spectral dynamic stiffness equation of the whole periodic structure}

In this section, the dynamic stiffness matrices for the individual plates are assembled into global matrix for the periodic plate structure in a manner similar with the FEM, except that the plates are connected along the boundary lines rather nodes. As a result, the equation of motion of the whole periodic plate structure can be obtained as

$$
\boldsymbol{f}^{g}=\boldsymbol{K}^{g} \boldsymbol{d}^{g}
$$

where $\boldsymbol{K}^{g}$ is the global spectral dynamic stiffness matrix, $\boldsymbol{d}^{g}$ and $\boldsymbol{f}^{g}$ are the displacement and force vectors in the global coordinate system.

\subsection{Forced vibration excitation load}

In this section, the application of the external excitation for the forced vibration is introduced. The force is assumed to be harmonically varying dynamic forces with the intensity of constant values.

Without loss of generality, the external normal force is located at boundary line 1 (as depicted in Fig. 4(a)) in this paper. Therefore, in the force vector $\boldsymbol{f}$, nonzero values only appear the vector $\boldsymbol{V}_{1}$ of $f_{1}$ as follow

$$
\boldsymbol{f}=\left[\boldsymbol{f}_{1}, \mathbf{0 , 0 , 0}\right]^{T}, \quad \boldsymbol{f}_{1}=\left[\boldsymbol{V}_{1}^{0}, \boldsymbol{V}_{1}^{1}, \mathbf{0 , 0}\right]^{T}
$$

According to the modified Fourier series given in Eq. (14) and the relation of $\boldsymbol{f}$ and $\boldsymbol{f}^{k j}$ given in Eq. (16), the shear force vectors $\boldsymbol{V}_{1}^{0}$ and $\boldsymbol{V}_{1}^{1}$ can be expressed as

$$
\begin{aligned}
& \boldsymbol{V}_{1}^{0}=\boldsymbol{V}_{a}^{00}+\boldsymbol{V}_{a}^{10} \\
& \boldsymbol{V}_{1}^{0}=\boldsymbol{V}_{a}^{01}+\boldsymbol{V}_{a}^{11}
\end{aligned}
$$

where

$$
\boldsymbol{V}_{a}^{k j}=\left[V_{a j 0}, V_{a j 1}, \cdots, V_{a j n}, \cdots\right]^{T}
$$

In which, the Fourier coefficients of the boundary conditions $V_{a j n}$ are determined as 


$$
V_{a j n}=\int_{-b}^{b} F(x, y) \frac{\Xi_{j}\left(\beta_{j n} y\right)}{\sqrt{\zeta_{j n} L}} d y
$$

where $F(x, y)$ is the external load located along the boundary line.

\subsection{Application of arbitrarily prescribed boundary conditions}

For a general Kirchhoff's thin plate, the governing equation is a forth order partial derivative function, and only two boundary constraints are required at each point of the boundary. Three major kinds of common boundary conditions may be specified on the plate boundary conditions.

- Kinematic boundary conditions : prescribed displacement and rotation (clamped edge)

$$
\begin{array}{ll}
w(\boldsymbol{r})-\bar{w}(\boldsymbol{r})=0, & \boldsymbol{r} \in B \\
\phi(\boldsymbol{r})-\bar{\phi}(\boldsymbol{r})=0, & \boldsymbol{r} \in B
\end{array}
$$

- Mechanical boundary conditions: prescribed bending moment and shear force (free edge)

$$
\begin{array}{ll}
m(\boldsymbol{r})-\bar{m}(\boldsymbol{r})=0, & \boldsymbol{r} \in \mathcal{B} \\
v(\boldsymbol{r})-\bar{v}(\boldsymbol{r})=0, & \boldsymbol{r} \in \mathcal{B}
\end{array}
$$

- Mixed boundary conditions: prescribed displacement and bending moment (simply supported edge)

$$
\begin{array}{ll}
w(\boldsymbol{r})-\bar{w}(\boldsymbol{r})=0, & \boldsymbol{r} \in \mathcal{B} \\
m(\boldsymbol{r})-\bar{m}(\boldsymbol{r})=0, & \boldsymbol{r} \in \mathcal{B}
\end{array}
$$

where $B$ represents boundaries of the plate. $\bar{w}(\boldsymbol{r}), \bar{\phi}(\boldsymbol{r}), \bar{v}(\boldsymbol{r})$ and $\bar{m}(\boldsymbol{r})$ are the prescribed displacement, normal rotation, shear force and bending moment respectively, which are set as zero vectors in the following procedures for simplicity. The boundary conditions are applied to the S-DSM by eliminating the rows and columns corresponding to the potential zero elements appeared in the displacement vector $\boldsymbol{d}$. 


\section{Numerical results}

In this part, the S-DSM is used to analyze the band gap properties appeared in the dynamic responses of the periodic plate structures. Firstly, an individual plate structure is adopted to validate the convergence and accuracy of the S-DSM. Then the vibration band gap properties in the periodic plate structures are mainly investigated by a number of numerical examples. To further study the accuracy and efficiency of the present method, the computational efficiency study is performed at last. For convenience, in the following, referring to the classical boundary conditions, F, S and C denote free, simply and clamped supported boundaries respectively.

\subsection{Individual plate structure}

In this section, the dynamic responses of the individual plate structure as shown in Fig. 4(a) are calculated. The structure and material parameters for the plate are taken as $a=0.2 \mathrm{~m}, b=0.2 \mathrm{~m}$, and the thickness $h=0.01 \mathrm{~m}$, the Young's modulus $E=200 \mathrm{GPa}$, the Poisson ratio $v=0.3$ and the mass density $\rho=7800 \mathrm{~kg} / \mathrm{m}^{3}$. Two parallel boundaries along $y= \pm b$ are supposed to be free, clamped supported and simply supported, and the other two boundaries which $x= \pm a$ are free. A point force $F=F_{0} \mathrm{e}^{\mathrm{i} \omega t}$ is applied on point $\mathrm{M}_{1}(0,-a)$, and $F_{0}$ is the amplitude of the force. In addition, the response point is located at $\mathrm{M}_{2}(0, a)$. A Matlab computer program is used to validate the S-DSM.

Since a limited number of terms in the general solution must be used for an actual calculation, the proposed solution in Eq. (13) should be truncated into finite terms. Thus, the convergence rate and computational efficiency should be regarded as the primary criterion of the present method. The convergence of solution for the individual plate structure with CFCF and SFSF boundary conditions are illustrated in Figs. 5 and 6, respectively. The number $N$ in the legend represents the truncated number for the $m$ and $n$ in Eq. (15), which indicates the number of the terms of the modified Fourier 
basis functions. It is clear that the results converge rapidly as the truncated number increases both

for CFCF and SFSF boundary conditions. Even when the truncated number is selected as 3, the convergence can be guaranteed in frequency range from 0 to $3000 \mathrm{~Hz}$. Furthermore, the calculation results are nearly identical when the truncated numbers $M$ and $N$ are fixed at 5 and 7 . Figs. 7 and 8 , respectively, show the comparisons of the dynamic responses of the individual plate structure under CC and SS boundary conditions. Good agreement of results obtained by the FEM and S-DSM models is obtained in lower frequency range. As the frequency increases, deviations appear between the results of the FEM with a coarse mesh $(10 \times 10)$ and the S-DSM. Nevertheless, the results of the finer meshed FEM $(20 \times 20)$ model show superior agreement with those of the S-DSM which can prove that the convergence of FEM towards to S-DSM. It also indicates that, if more accurate FEM results are desired in high frequency range, much finer mesh should be guaranteed firstly. In conclusion, the present method can deal with problems of higher frequency with small truncated number and keep excellent accuracy in contrast with the FEM.

\subsection{Periodic plate structure}

In this section, the vibration band gap properties of the periodic plate structures shown in Fig. 4(b) are investigated. Firstly, several examples are implemented to validate the present method by comparing with the FEM. Additionally, a systematic parameter study is conducted to research the effects of boundary conditions, materials and dimensional properties of the unit plate on the band gap behaviors. In the following procedures, the number of the unit plate is fixed equal to 15 and the periodic structure with epoxy as material $A$ and steel as material $B$ are investigated without special instructions. The physical parameters of the materials used in following examples are listed in Table 1. 


\subsubsection{Validation}

In order to further validate the ability of the present method for solving the forced vibration problems of the periodic plate structures, several numerical examples are given in this subsection. A vertical point force $F=F_{0} e^{i \omega t}$ is applied at $x=-a, y=0$ as depicted in Fig. 4(b), where $a=b=0.2$ $\mathrm{m}$ and $F_{0}=10 \mathrm{~N}$. The truncated number for the modified Fourier series is selected as $M=N=5$. With regard to the periodic plate structure, only boundary lines parallel to the $y$ axis are taken into consideration and those which are parallel to the $x$ axis are considered as free or coupled with each other. For example, for the CS boundary condition, the first letter C indicates that the boundary line along $x=b$ are clamped supported, and the second letter $\mathrm{S}$ indicates the boundary line along $x=-b$ are simple supported.

Fig. 9 presents the comparison of the dynamic responses calculated by the S-DSM and FEM for the periodic plate structure with CC boundary condition. For comparison, two types of mesh ( $8 \times 8$ and $16 \times 16$ for a unit plate) are selected for the FEM. One can see that the present solutions are in good agreement with those obtained by the FEM. Meanwhile, it is noted that the solutions of $16 \times 16$ mesh FEM model are closer to the S-DSM results compared with those of the $8 \times 8$ mesh FEM model, particularly in high frequency scopes. That is, the FEM results converge to the S-DSM results.

In addition, Figs.10-13 give the dynamic responses of the periodic plates under CS, SS, CF and SF boundary conditions. The results of the S-DSM and FEM agree well with each other. Also, the band gaps can be observed obviously in the response curves for all the boundary conditions, especially in the lower frequency range. However, compared with response curves of the CF and SF boundary conditions, more and wider band gaps appear in the response curves of the CS and SS boundary conditions. In Fig. 12, only three obvious band gaps can be observed, and barely no band 
gap appears in the frequency range from 700-1500 Hz. Although, many band gaps can be observed

in the lower frequency rage in Fig. 13, but the band gaps are very narrow and only one obvious band gap arises from $600-1500 \mathrm{~Hz}$. Therefore, the F boundary condition is not conductive to the formulation of band gaps.

Figs. 14 and 15 show the transverse responses at each unit plate in the periodic plate structure for frequencies located at stop band and pass band respectively. In Fig. 14, the selected five frequencies are located in the first five stop bands respectively, i.e., $f=206,306,476,641$ and 866 Hz. On the whole, the vibration amplitudes decrease obviously as the number of the plate increases. The ratio of the vibration amplitudes of the first and the last plate is considerably large. Therefore, the vibration cannot propagate through the whole periodic plate when the harmonic frequency is located at the stop bands. On the contrary, Fig. 15 shows the responses for each unit plate for frequencies which are located at the pass bands, i.e., $f=166,246,401,561$ and $756 \mathrm{~Hz}$. Despite the ups and downs, one can observe that the excitation under those frequencies at the left of the structure all propagate through the whole periodic structure.

\subsubsection{Effect of material parameters}

In this subsection, parametric investigations are implemented to study the effects of the material parameters on the band gap behaviors. For simplicity's sake, only CC and SS boundary conditions are taken into consideration. Figs. 16 and 17 depict the comparisons of the dynamic responses for periodic plates with different materials for the unit plate. In the figures, one can see that there is almost no band gap in the response curves for copper as material $A$ and steel as material $B$. When the material $B$ is aluminum, several small band gaps appear in the response curves. Furthermore, the response curves have clearer drops inside the band gaps when the materials $A$ and $B$ are epoxy and steel respectively. From the analysis, it can be concluded that the larger the 
differences of the properties between materials $A$ and $B$ the more obvious band gaps appear in the

response curves.

In practical engineering, the effects of structural damp are always non-negligible. In this work, structural damping is introduced in the present formulation by replacing Young's modulus $E$ by a complex one $E=E(1+i \eta)$, where $\eta$ is the material loss factor. The effects of damping on the vibration responses of the periodic structure under SS boundary condition are illustrated in Fig. 18. It can be observed from Fig. 18 that with the introduction of the structural damping the locations of the band gaps remain unchanged. The comparison of the vibration responses of the damped and undamped structures indicates that the structural damping suppresses the amplitudes of the resonant peaks in the pass bands, especially in high frequency range. Meanwhile, the structure damping has little influence on the vibration amplitudes for non-resonant vibration.

\subsubsection{Effect of geometrical parameters}

In the following procedures, the band gap properties of periodic structures composed of unit plate with different geometric dimensions are studied. It is assumed that both the materials $A$ and $B$ of the unit plate in Fig. 4(b) are uniformly chosen as epoxy. The symbols $h_{1}$ and $h_{2}$ represent the thicknesses of plate $A$ and $B$. In the calculations, $h_{1}$ increases from $0.008 \mathrm{~m}$ to $0.012 \mathrm{~m}$ with $h_{2}$ fixed at $0.005 \mathrm{~m}$. The corresponding dynamic responses for the periodic structures with different thicknesses for unit plate are given in Figs. 19 and 20. As expected, more obvious band gaps appear as the $h_{1}$ becomes larger. However, it is a remarkable fact that, only when the ratio of $h_{1}$ and $h_{2}$ becomes very large, e.g. $h_{1} / h_{2}=2.4$, obvious band gaps turn up in the dynamic response curves. This may be unacceptable in practical engineering. So, it's pretty obvious that the band gap properties can not only rely on the difference of geometrical dimensions between the plates. Therefore, the superposed effects of both the geometrical dimension and material property on the 
band gap of the periodic plate structures are considered. For simplicity, only SS boundary condition

is taken into consideration here. For comparison, Fig. 21(a) shows the dynamic responses of the periodic plate structure with different thicknesses and identical material for the unit plate and Fig. 21(b) shows the dynamic responses of the periodic plate structure with different thicknesses and different material properties. As one can see in Fig. 21 (a), no band gap appears in the dynamic response curves as $h_{1}$ increases. On the opposite, in Fig. 21(b), the band gaps become more and wider in the responses curves as $h_{1}$ changes with only a small drop. In conclusion, when the periodic structure is composed of plate with different materials it becomes much more sensitive to the dimensional variation of the unit plate. Small change can lead to significant effects on the band gap properties which has good reference to practical engineering.

Fig. 22 shows the frequency ranges of the first four stop bands with different ratio between the thickness and width of the unit plate. In the figures, the grey shaded parts represent the stop band of the vibration response and the corresponding colorless parts indicate the pass bands. When the ratio increases from $1 / 50$ to $1 / 35$, the bandwidth of the grey shaded part becomes larger but not particularly evident. On the other hand, the relative frequency ranges of the stop bands increase obviously as the ratio of $h / 2 a$ increases gradually. In conclusion, the thickness of the plate has not much influence on the bandwidth of the band gaps, but it affects the range of band gaps evidently.

Figs. 23 and 24, respectively, show the comparisons of dynamic responses for periodic structures with different unit plate numbers. In the calculation, the geometric dimensions of the individual plates are consistent and the matrials of the structures are composed of epoxy and steel. It is clear that the frequency ranges of the band gaps have no change as the number of the plates increases from 5 to 10 to 15 . But as the number of the unit plate inreases, the stop bands become 
deeper. As a result, the number of the unit cell in the periodic structure has no effect on the positions

of the band gaps but has significant effects on the depths of the band gaps.

\subsubsection{Computational efficiency}

In order to further validate the accuracy and efficiency of the S-DSM, a computational efficiency study is performed. The dynamic responses of the periodic structure are calculated by the S-DSM with different truncated numbers and by the FEM with different mesh sizes, separately. Without loss of generality, only two response points are selected as illustration, i.e., one point at the middle and another on at the end of the structure. For comparison, the averaged error $\langle\varepsilon\rangle$ on forced responses of the two response points can be obtained as follow

$$
\langle\varepsilon\rangle=\frac{1}{n_{r p}} \sum_{j=1}^{n_{r p}} \varepsilon_{j}
$$

where

$$
\varepsilon_{j}=\frac{\left\|w(\boldsymbol{r})-w_{\mathrm{ref}}(\boldsymbol{r})\right\|}{\left\|w_{\mathrm{ref}}(\boldsymbol{r})\right\|}
$$

where $n_{r p}=2$. As references, the results of an S-DSM model with the truncated number $M=N=9$ and an FEM model with mesh size equal to 0.005 are both taken into consideration. In practical calculation, the frequency range of the responses is from 200 to $300 \mathrm{~Hz}$ and the substep is selected as $0.5 \mathrm{~Hz}$.

Fig. 25 plots the averaged relative prediction error of the normal displacement as a function of the calculation time. In Fig. 25(a), the results of an S-DSM model are adopted as the reference. Under the same averaged relative error, the S-DSM takes less time. Obviously, the S-DSM shows much higher computational efficiency. In Fig. 25(b), the results of the FEM model are selected as the reference and the S-DSM also performs better than the FEM. In addition, the S-DSM is 
implemented as a research code in Matlab environment while the FEM is conducted in an optimized commercial program.

The S-DSM exhibits the excellent computational efficiency. To obtain the same computation error value, the computational time of the S-DSM is much less than that of the FEM.

\section{Conclusion}

In this paper, the band gap properties of the periodic plate structures with general boundary conditions are studied by using the S-DSM. In this paper, firstly, the computational accuracy and convergence rate of the S-DSM are validated. Then, numerous numerical examples are presented to investigate the band gap properties of the periodic plate structures with different boundary conditions. From the numerical results, the following conclusions can be drawn:

(1) The S-DSM is employed to solve the forced vibration problems of the periodic plate structures and study the band gap properties appearing in the dynamic responses. Good agreement has been achieved by comparing the present results with those obtained by the FEM.

(2) The material properties have great effects on the band gap behaviors of the periodic plate structures. Basically, the greater differences between the materials the more obvious band gap appears.

(3) Only large scale differences in geometrical dimensions of the unit plate can lead to obvious band gap phenomenon. However, if the material properties of the unit plate are different, a small deviation of the geometrical dimensions can affect the width and location of the band gaps evidently. 
(4) The structural damping only affects the magnitude of the renascent peaks in the pass

band, especially in high frequency domain. Besides, the locations of the band gaps are hardly influenced.

This paper provides a detailed numerical investigation into the band gap properties of the periodic structures. Numerous numerical results provided in this paper can be used as reference for further researches and engineering design. In the future research, the S-DSM can be extended to investigate the vibration characteristic of periodic rib-stiffened plate structures, periodic sandwich panels with corrugated cores and so on.

\section{Acknowledgement}

The authors gratefully acknowledge the financial support from the National Science Foundation of China (Nos. 51279035 and 51175098) and the Fundamental Research Funds for the Central Universities (Nos. HEUCFQ1401 and HEUCF160304).

\section{References}

[1] Mead D J. A general theory of harmonic wave propagation in linear periodic systems with multiple coupling. J Sound Vib 1973; 27(2):235-260.

[2] Mead DJ. Wave propagation in continuous periodic structures: research contributions from Southampton 1964-1995. J Sound Vib 1996; 190(3):495-524.

[3] Kushwaha MS, Halevi P, Dobrzynski L, Djafari-Rouhani B. Acoustic band structure of periodic elastic composites. Phys Rev Lett 1993; 71(13):2022-2025. 
[4] Richards D, Pines D J. Passive reduction of gear mesh vibration using a periodic drive shaft. J Sound Vib 2003; 264(2):317-342.

[5] Yu DL, Liu YZ, Qu J, Zhang HG, Liu ZM. Experimental and theoretical research on the vibrational gaps in two-dimensional three-component composite thin plates. Chin Phys Lett 2005; 22(8):1958-1960.

[6] Duhamel D, Mace BR, Brennan M J. Finite element analysis of the vibrations of waveguides and periodic structures. J Sound Vib 2006; 294(s1-2):205-220.

[7] Wang G, Wen X, Wen J, Liu YZ. Quasi-one-dimensional periodic structure with locally resonant band gap. J Appl Mech 2006; 73(1): 167-170.

[8] Chen Y, Li J, Zhou JD, Huang T, Zhou MG, Yu DY. Phononic first band gap of quaternary layered periodic structure with the lumped-mass method. Shock Vib 2014; 2014(2):1-9.

[9] Yan ZZ, Wang YS. Wavelet-based method for computing elastic band gaps of one-dimensional phononic crystals. Sci China 2007; 50(05):622-630.

[10] Yan ZZ, Wang YS. Wavelet-based method for calculating elastic band gaps of two-dimensional phononic crystals. Phys Rev B 2006; 74(22):4070-4079.

[11]Richards D, Pines D J. Passive reduction of gear mesh vibration using a periodic drive shaft. J Sound Vib 2003; 264(2):317-342.

[12] Yan ZZ, Wang YS. Calculation of band structures for surface waves in two-dimensional phononic crystals with a wavelet-based method. Phys Rev B 2008; 78(9): 094306

[13]Xiang HJ, Shi ZF. Analysis of flexural vibration band gaps in periodic beams using differential quadrature method. Comput Struct 2009; 87(23):1559-1566. 
[14]Norris R, Nieva P, Hamel J. Analytical analysis of a discrete MEMS diatomic mass-spring phononic band gap crystal for vibration stabilization applications. IEEE Sensors 2008; 506-509.

[15]Ruzzene M, Baz A. Control of wave propagation in periodic composite rods using shape memory inserts. J Vib Acoust 1999; 122(2):151-159.

[16] Marino MA, Berardo A. Attenuation and localization of wave propagation in periodic rods using shape memory inserts. Smart Mater Struct 2000; 9(6):805-816(12).

[17] Yu DL, Liu YZ, Zhao HG, Wang G, Qiu J. Flexural vibration band gaps in Euler-Bernoulli beams with locally resonant structures with two degrees of freedom. Phy Rev B 2006; 73(6), 064301.

[18]Xiang HJ, Shi ZF. Analysis of flexural vibration band gaps in periodic beams using differential quadrature method. Comput Struct 2009; 87(23):1559-1566.

[19] Olhoff N, Niu B, Cheng G. Optimum design of band-gap beam structures. Int J Solids Struct 2012; 49(22):3158-3169.

[20]Hussein MI, Hamza K, Hulbert GM, Scott RA, Saitou K. Multiobjective evolutionary optimization of periodic layered materials for desired wave dispersion characteristics. Struct Multidiscip O 2006; 31(1):60-75.

[21]Ribeiro P, Stoykov S. Forced periodic vibrations of cylindrical shells in laminated composites with curvilinear fibres. Compos Struct 2015; 131:462-478.

[22] Tomczyk BA Non-asymptotic model for the stability analysis of thin biperiodic cylindrical shells. Thin Wall Struct 2007; 45(10-11):941-944.

[23] Tomczyk B. On the modelling of thin uniperiodic cylindrical shells. J Theor App Mech 2003; 41(4): 755-774. 
[24] Shen HJ, Wen JH, Yu DL, Wen XS. The vibrational properties of a periodic composite pipe in 3D space. J Sound Vib 2009; 328(s1-2):57-70.

[25] Wen JH, Shen HJ, Yu DL, Wen XS. Theoretical and experimental investigation of flexural wave propagating in a periodic pipe with fluid-filled loading. Chinese Phys Lett 2010; 27(11):133-136.

[26] Sorokin SV, Ershova OA. Analysis of the energy transmission in compound cylindrical shells with and without internal heavy fluid loading by boundary integral equations and by Floquet theory. J Sound Vib 2006; 291(1-2):81-99.

[27] Shen HJ, Wen JH, Paidoussis MP, Yu DL, Asgari M, Wen XS. Control of sound and vibration for cylindrical shells by utilizing a periodic structure of functionally graded material. Phys Lett A 2012; 376(45):3351-3358.

[28] Shen HJ, Wen JH, Yu DL, Asgari M, Wen XS. Control of sound and vibration of fluid-filled cylindrical shells via periodic design and active control. J Sound Vib 2013; 332(18):4193-4209.

[29] Sigalas MM, Economou EN. Elastic waves in plates with periodically placed inclusions. J Appl Phys 1994; 75(6):2845-2850.

[30]Langley RS, Smith JRD, Fahy FJ. Statistical energy analysis of periodically stiffened damped plate structures. J Sound Vib 1997; 208(3):407-426.

[31]Wang JW, Wang G, Wen JH, Wen XS. Flexural vibration band gaps in periodic stiffened plate structures. Mech 2012; 18(2):186-191.

[32] Wu ZJ, Li FM, Wang YZ. Study on vibration characteristics in periodic plate structures using the spectral element method. Acta Mech 2013; 224(5):1089-1101. 
[33] Wu ZJ, Li FM, Wang YZ. Vibration band gap properties of periodic Mindlin plate structure using the spectral element method. Meccanica 2014; 49(3):725-737.

[34]Banerjee JR, Jackson DR. Free vibration of a rotating tapered Rayleigh beam: A dynamic stiffness method of solution. Comput Struct 2013; 124(8):11-20.

[35]Banerjee JR, Cheung CW, Morishima R, Perera M, Njuguna J. Free vibration of a three-layered sandwich beam using the dynamic stiffness method and experiment. Int $\mathbf{J}$ Solids Struct 2007; 44(22):7543-7563.

[36]Li J, Hua HX. Dynamic stiffness analysis of laminated composite beams using trigonometric shear deformation theory. Compos Struct 2009; 89(3):433-442.

[37] Wittrick WH, Williams FW. Buckling and vibration of anisotropic or isotropic plate assemblies under combined loadings. Int J Mech Sci 1974; 16(4): 209-239.

[38]Fazzolari FA, Boscolo M, Banerjee J R. An exact dynamic stiffness element using a higher order shear deformation theory for free vibration analysis of composite plate assemblies. Compos Struct; 2013, 96: 262-278.

[39]Liu X, Banerjee JR. Free vibration analysis for plates with arbitrary boundary conditions using a novel spectral-dynamic stiffness method. Comput Struct 2015; 164:108-126.

[40]Liu X, Banerjee JR. An exact spectral-dynamic stiffness method for free flexural vibration analysis of orthotropic composite plate assemblies - Part I: Theory. Compos Struct 2015; $132: 1274-1287$.

[41]Liu X, Banerjee JR. An exact spectral-dynamic stiffness method for free flexural vibration analysis of orthotropic composite plate assemblies - Part II: Applications. Compos Struct $2015 ; 132: 1288-1302$ 
[42]Liu X, Kassem HI, Banerjee J R. An exact spectral dynamic stiffness theory for composite plate-like structures with arbitrary non-uniform elastic supports, mass attachments and coupling constraints. Compos Struct 2016; 142:140-154.

[43]Leissa AW. The free vibration of rectangular plates. J Sound Vib 1973; 31(3):257-93.

[44]Boyd JP. Chebyshev and Fourier spectral methods. 2ed. Dover Publications; 2001.

[45]Bracewell RN. The Fourier transform and its applications. 2ed ed. London: McGraw-Hill Book Company; 1978.

\section{List of Collected Table and Figures and Figure Captions}

Table 1. Physical parameters of the materials.

Fig.1 Arrangement of the periodic plate structure.

Fig.2 Rectangular plate.

Fig. 3 (a): Definition of the BC applied on the entire plate $\Omega$, (b): Definition of BC applied on boundaries $B_{a}$ and $B_{b}$.

Fig. 4. Type of load and response point of the individual plate structure.

Fig.5 Convergence of the S-DSM solutions for plate with CFCF boundary condition.

Fig.6 Convergence of the S-DSM solutions for plate with SFSF boundary condition.

Fig.7 Comparison of responses of plate with SFSF boundary condition by FEM and S-DSM.

Fig.8 Comparison of responses of plate with CFCF boundary condition by FEM and S-DSM. 
Fig. 9 Comparison of dynamic responses of the periodic plate structure with CC by the S-DSM and FEM.

Fig. 10 Comparison of dynamic responses of the periodic plate structure with CS by the S-DSM and FEM.

Fig. 11 Comparison of dynamic responses of the periodic plate structure with SS by the S-DSM and FEM.

Fig. 12 Comparison of dynamic responses of the periodic plate structure with CF by the S-DSM and FEM.

Fig. 13 Comparison of dynamic responses of the periodic plate structure with SF by the S-DSM and FEM.

Fig. 14 Vibration responses for each unit plate for frequency located at the stop band.

Fig. 15Vibration responses for each unit plate for frequency located at the pass band.

Fig.16 Comparison of frequency responses for periodic structure with different materials under CC boundary condition (Band gap rages: $-150-160 \mathrm{~Hz} ;-\cdot-191-224 \mathrm{~Hz}, 265-361 \mathrm{~Hz}, 429-507 \mathrm{~Hz}$, $807-1001 \mathrm{~Hz} ; \cdots \cdot \cdot 285-339 \mathrm{~Hz}, 451-489 \mathrm{~Hz}, 619-703 \mathrm{~Hz}, 865-959 \mathrm{~Hz})$.

Fig.17 Comparison of frequency responses for periodic structure with different materials under SS boundary condition (Band gap rages: $-374-397 \mathrm{~Hz} ;=-391-413 \mathrm{~Hz}, 479-585 \mathrm{~Hz}, 666-744 \mathrm{~Hz}$, 841-1045Hz; $\cdots . .303-337 H z, 393-417 \mathrm{~Hz}, 685-740 \mathrm{~Hz}, 901-1003 \mathrm{~Hz})$.

Fig .18 Damping effects on the vibration responses of the periodic plate structure.

Fig. 19 Comparisons of band gaps for periodic plate structure with different thickness under CC boundary conditions (Band gap rages: $-270-280 \mathrm{~Hz}, 365-385 \mathrm{~Hz}, 475-495 \mathrm{~Hz} ;-\cdot=162-171 \mathrm{~Hz}$, 295-323Hz, 515-559Hz; '....181-194Hz, 240-254Hz, 319-355Hz, 429-463Hz, 561-602Hz). 
Fig. 20 Comparisons of band gaps for periodic plate structure with different thickness under SS

boundary conditions (Band gap rages: _ none; $-\cdot-327-371 \mathrm{~Hz}, 721-801 \mathrm{~Hz} ; \cdots \cdot . .351-419 \mathrm{~Hz}$, $767-883 \mathrm{~Hz})$.

Fig. 21 The superposed influence of the geometrical and material properties on the band gaps.

Fig. 22 The frequency ranges of the first four stop bands of the periodic plate.

Fig. 23 Dynamic responses of periodic consisted of different number of unit cell under CC boundary condition. (Band gap rages: $389-438 \mathrm{~Hz}, 514-579 \mathrm{~Hz}, 686-800 \mathrm{~Hz}, 910-1078 \mathrm{~Hz}$, $1482-1772 \mathrm{~Hz})$

Fig. 24 Dynamic responses of periodic consisted of different number of unit cell under SS boundary condition(Band gap rages: $191-223 \mathrm{~Hz}, 265-361 \mathrm{~Hz}, 429-507 \mathrm{~Hz}, 587-715 \mathrm{~Hz}$, $809-999 \mathrm{~Hz}, 1133-1200 \mathrm{~Hz}, 1465-1557 \mathrm{~Hz})$.

Fig. 25 Relative error as function of the calculation time. 
Figures:

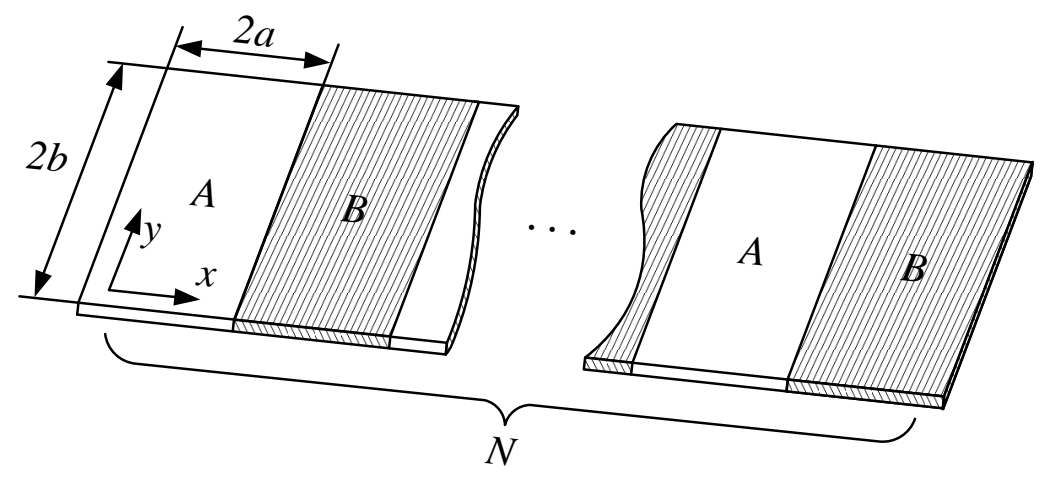

Fig.1 Arrangement of the periodic plate structure.

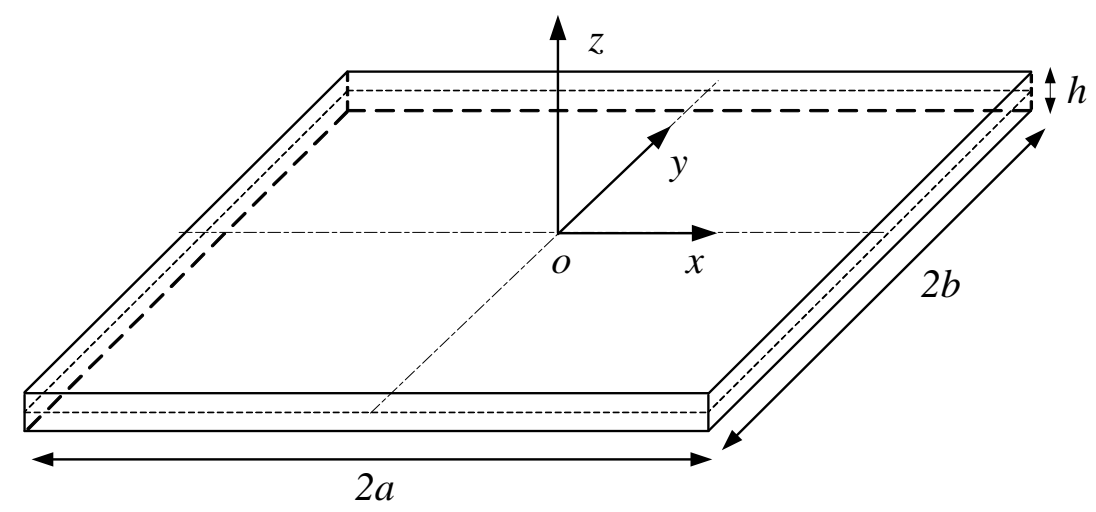

Fig.2 Rectangular plate. 


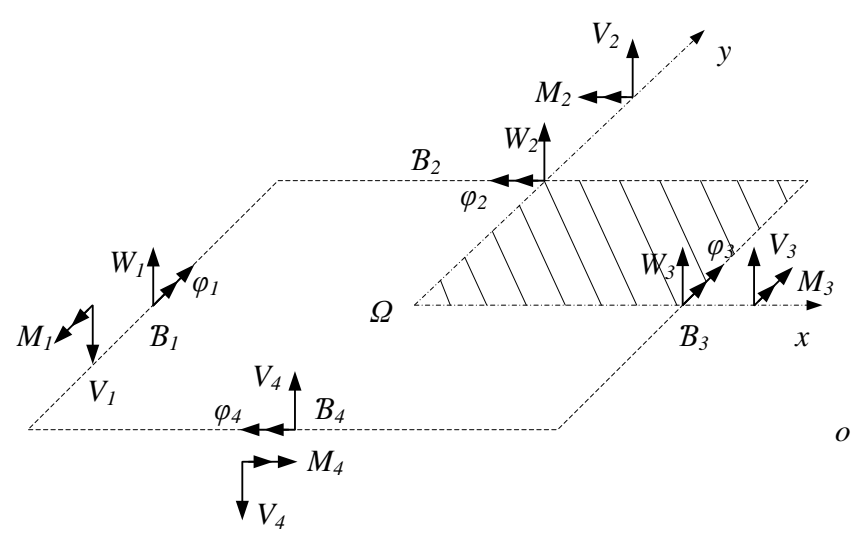

(a) BC applied to $\Omega$.

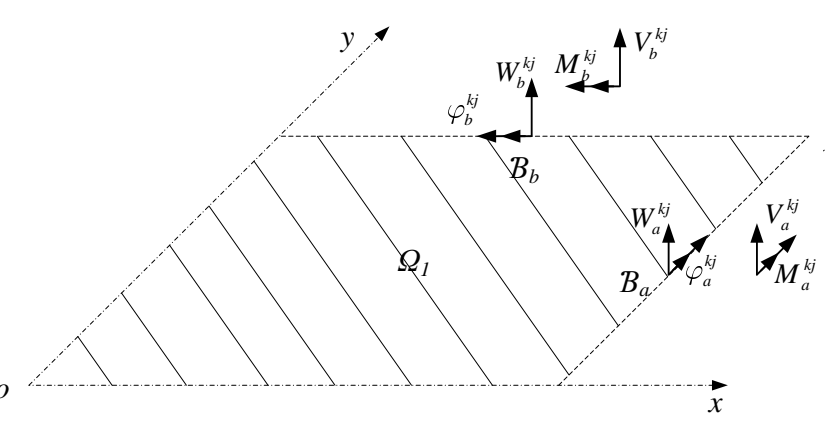

(b) BC applied to $\Omega_{1}$.

Fig. 3 (a): Definition of the BC applied on the entire plate $\Omega$, (b): Definition of BC applied on boundaries $B_{a}$ and $B_{b}$.

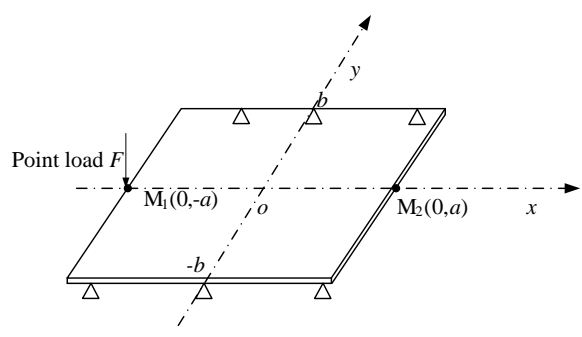

(a)

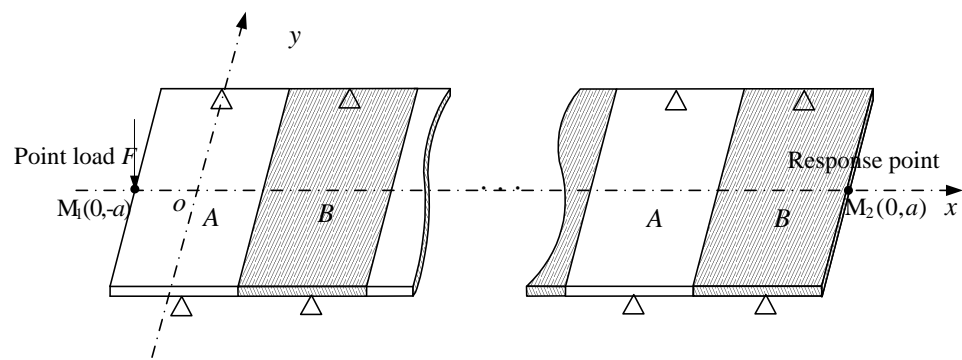

(b)

Fig. 4. Type of load and response point of the individual plate structure. 


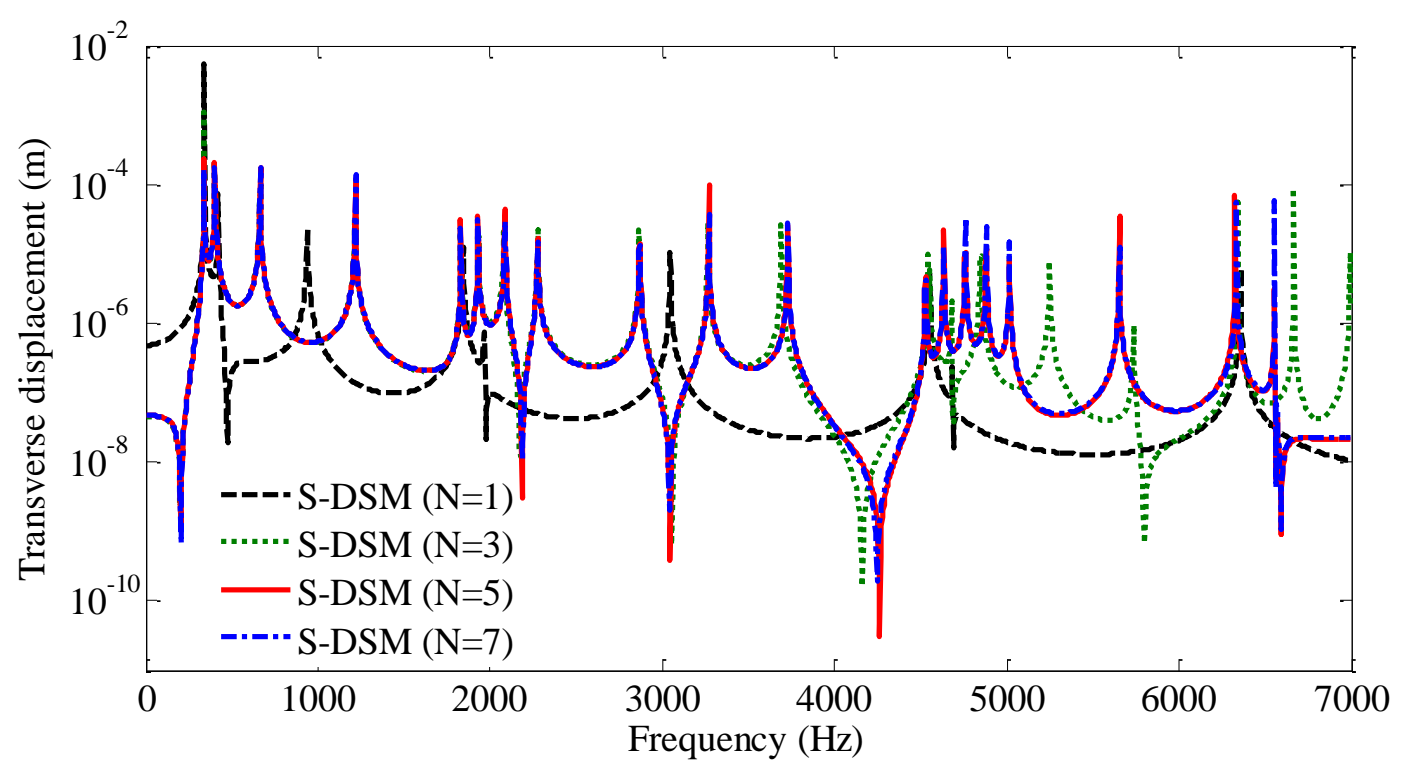

Fig.5 Convergence of the S-DSM solutions for plate with CFCF boundary condition.

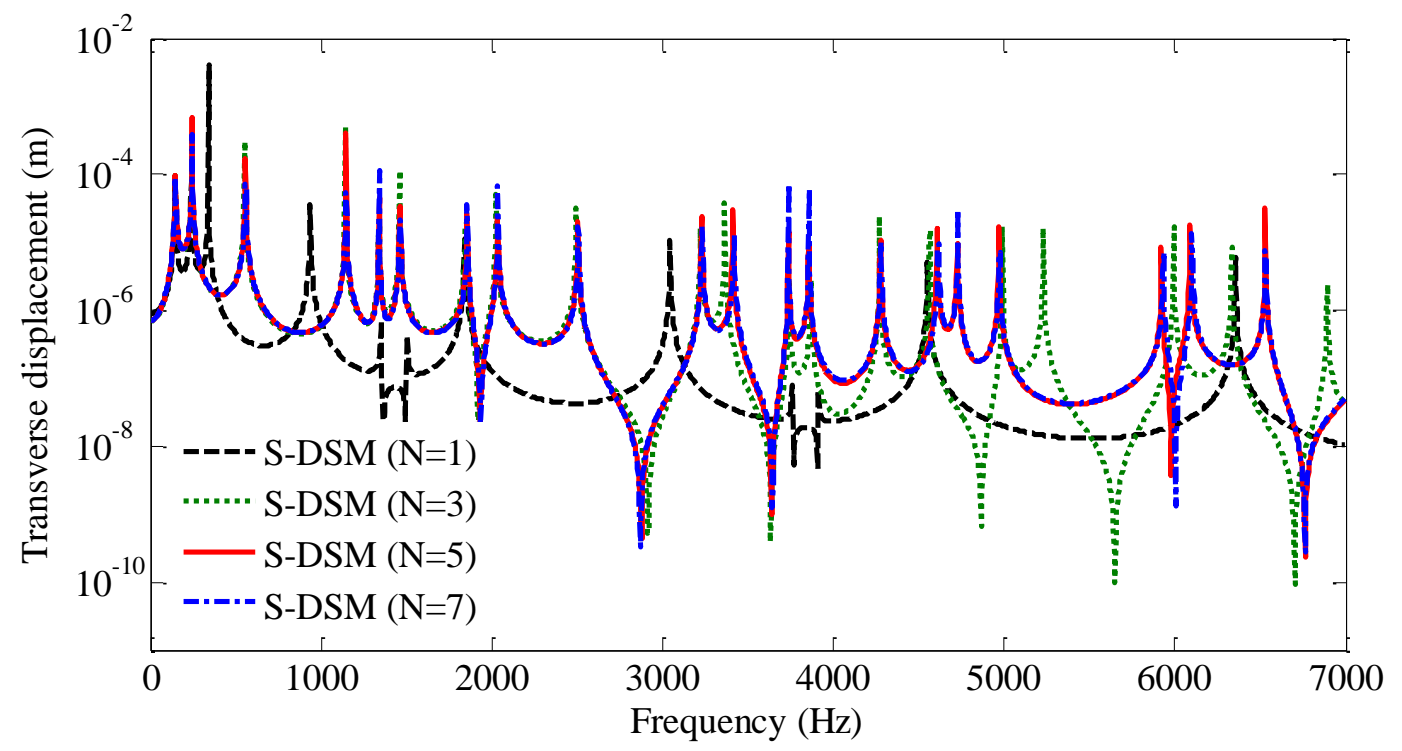

Fig.6 Convergence of the S-DSM solutions for plate with SFSF boundary condition. 


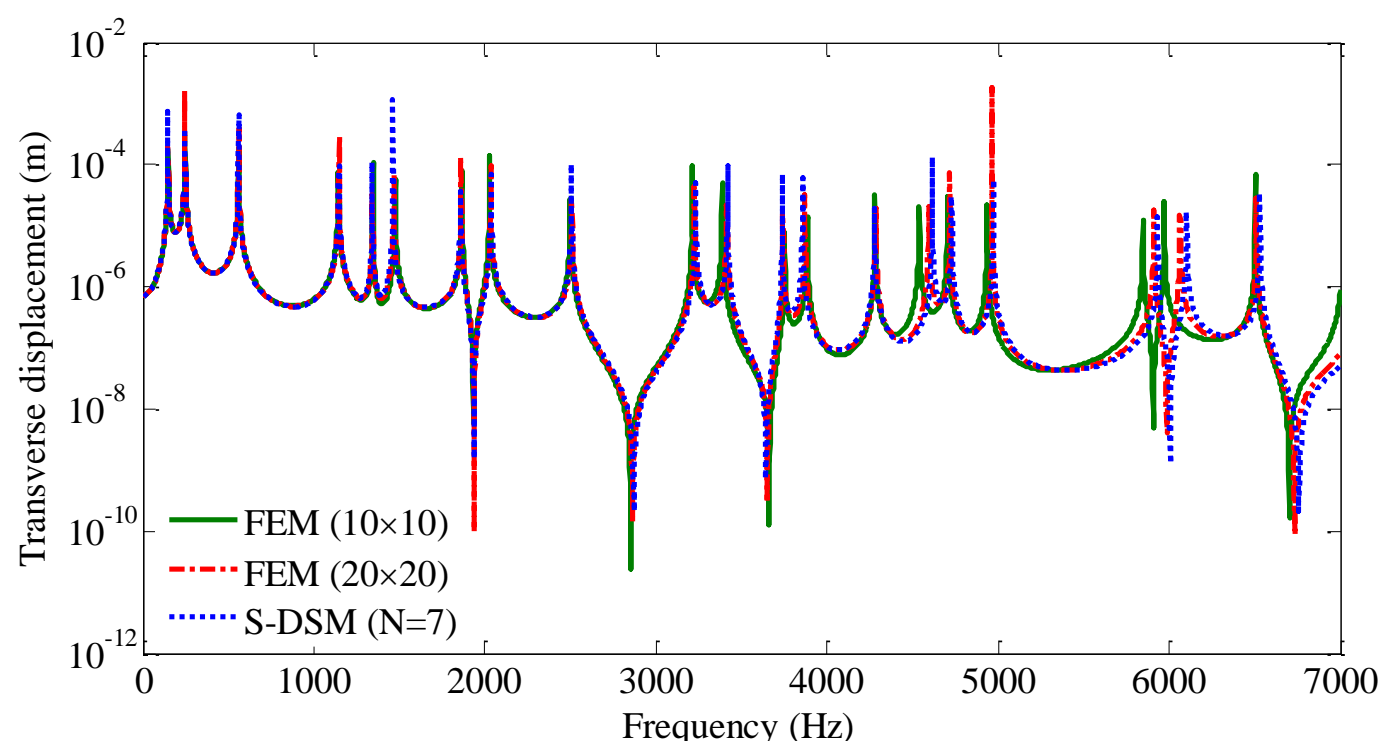

Fig.7 Comparison of responses of plate with SFSF boundary condition by FEM and S-DSM.

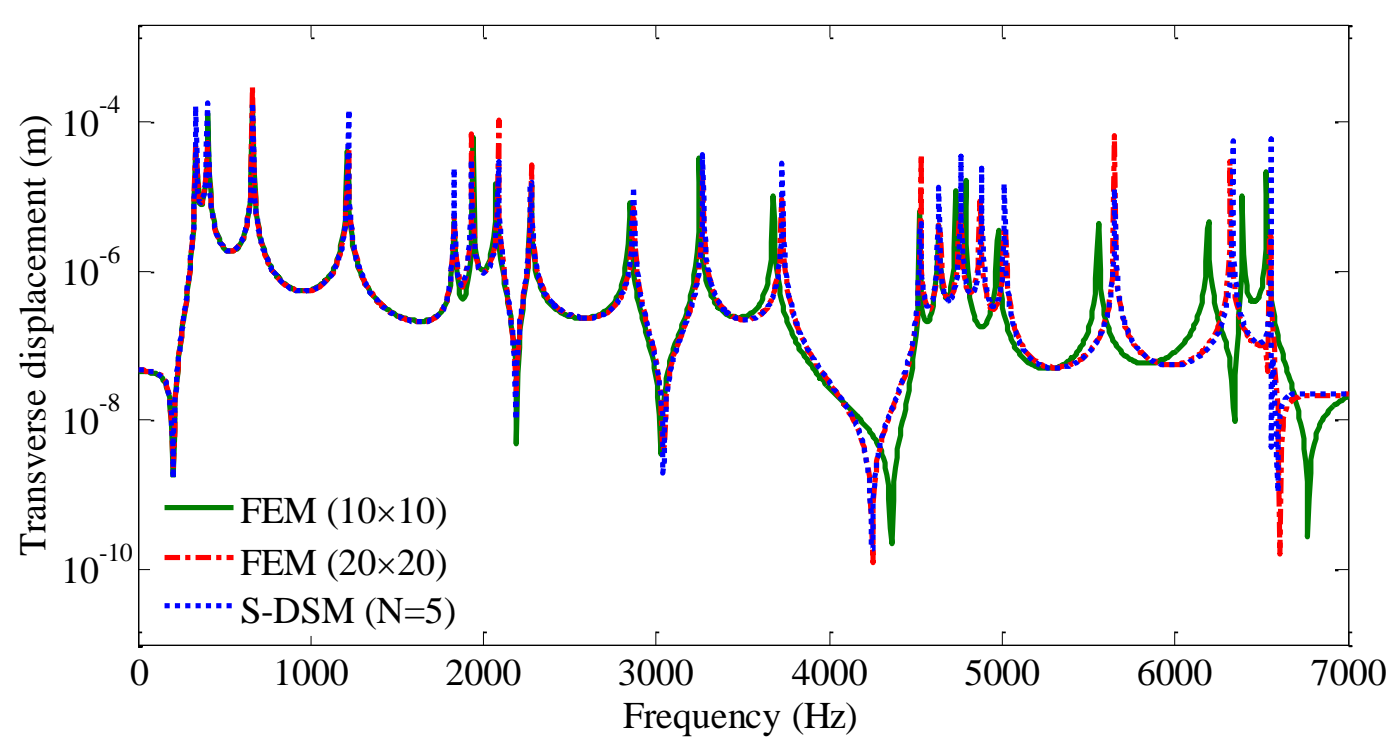

Fig.8 Comparison of responses of plate with CFCF boundary condition by FEM and S-DSM. 


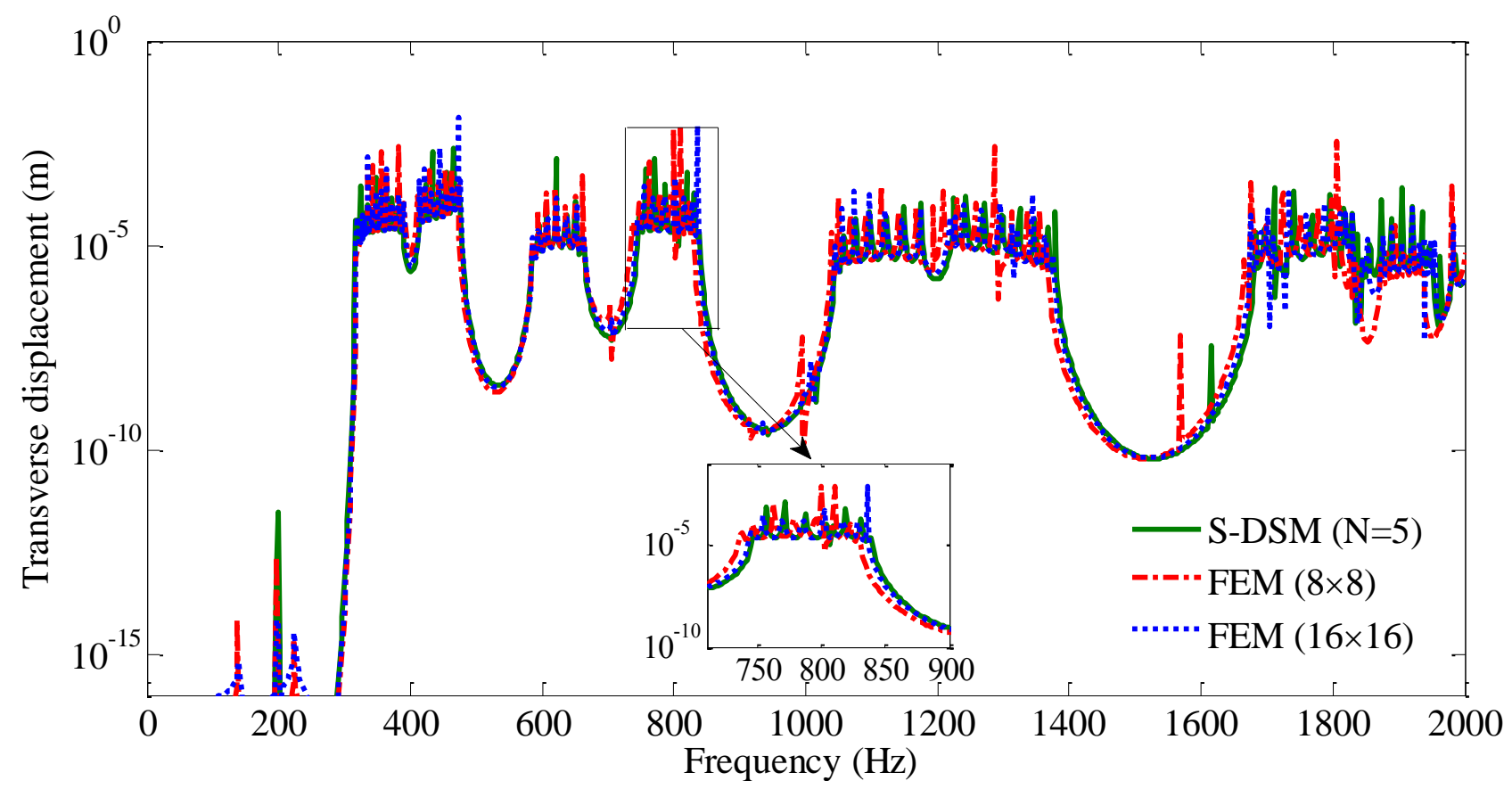

Fig. 9 Comparison of dynamic responses of the periodic plate structure with CC by the S-DSM and

FEM.

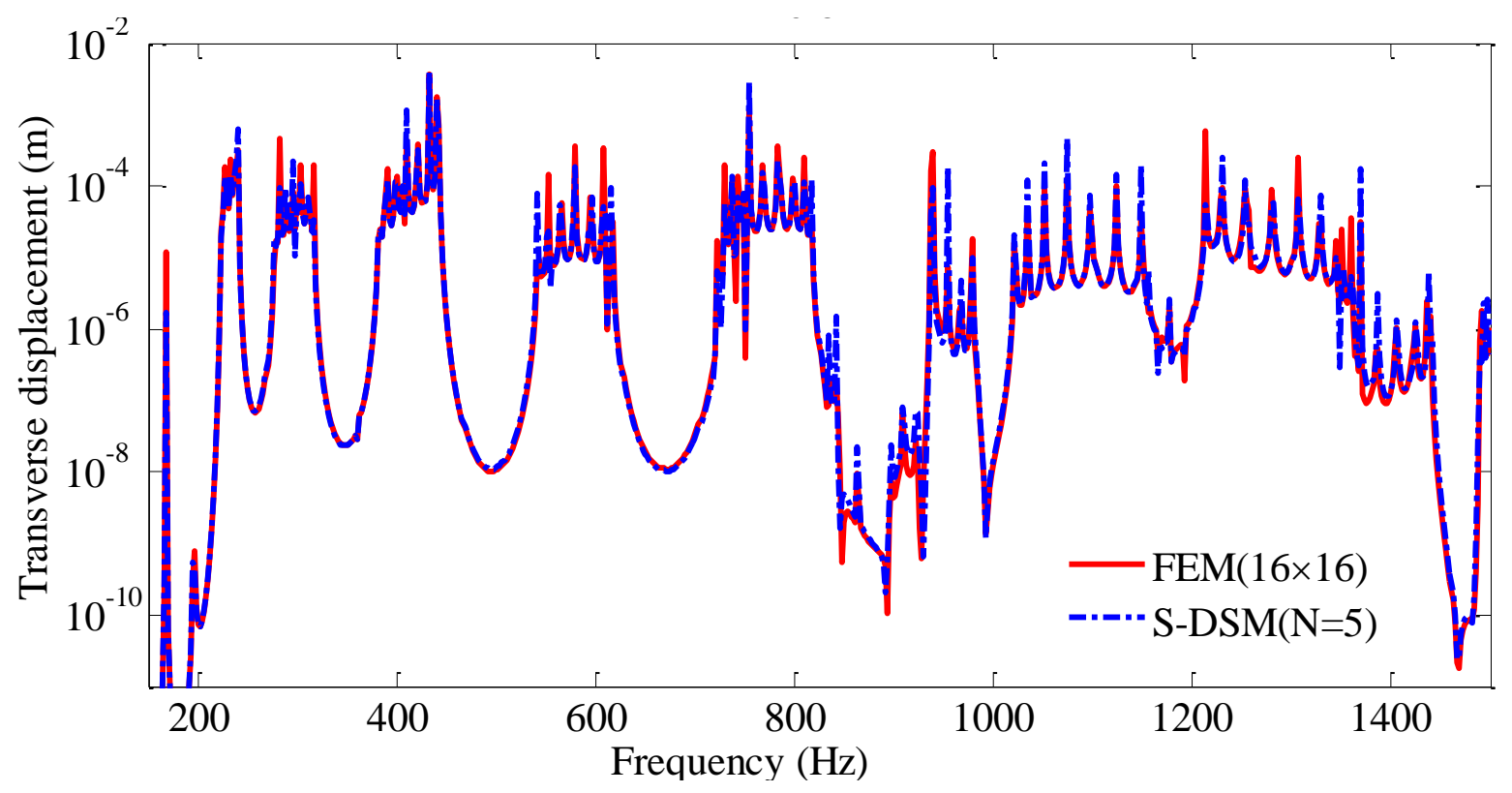

Fig. 10 Comparison of dynamic responses of the periodic plate structure with CS by the S-DSM and FEM. 


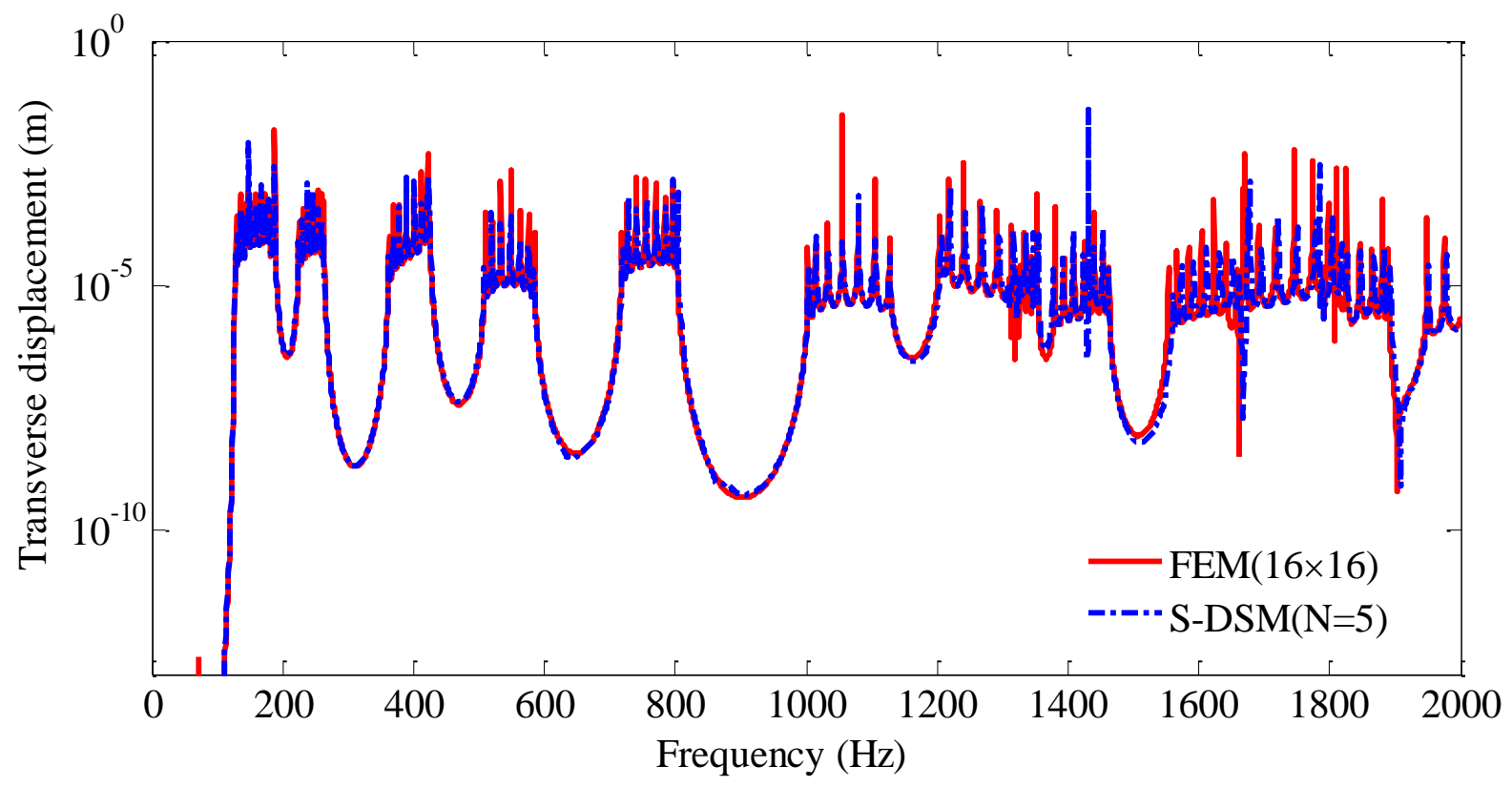

Fig. 11 Comparison of dynamic responses of the periodic plate structure with SS by the S-DSM and FEM.

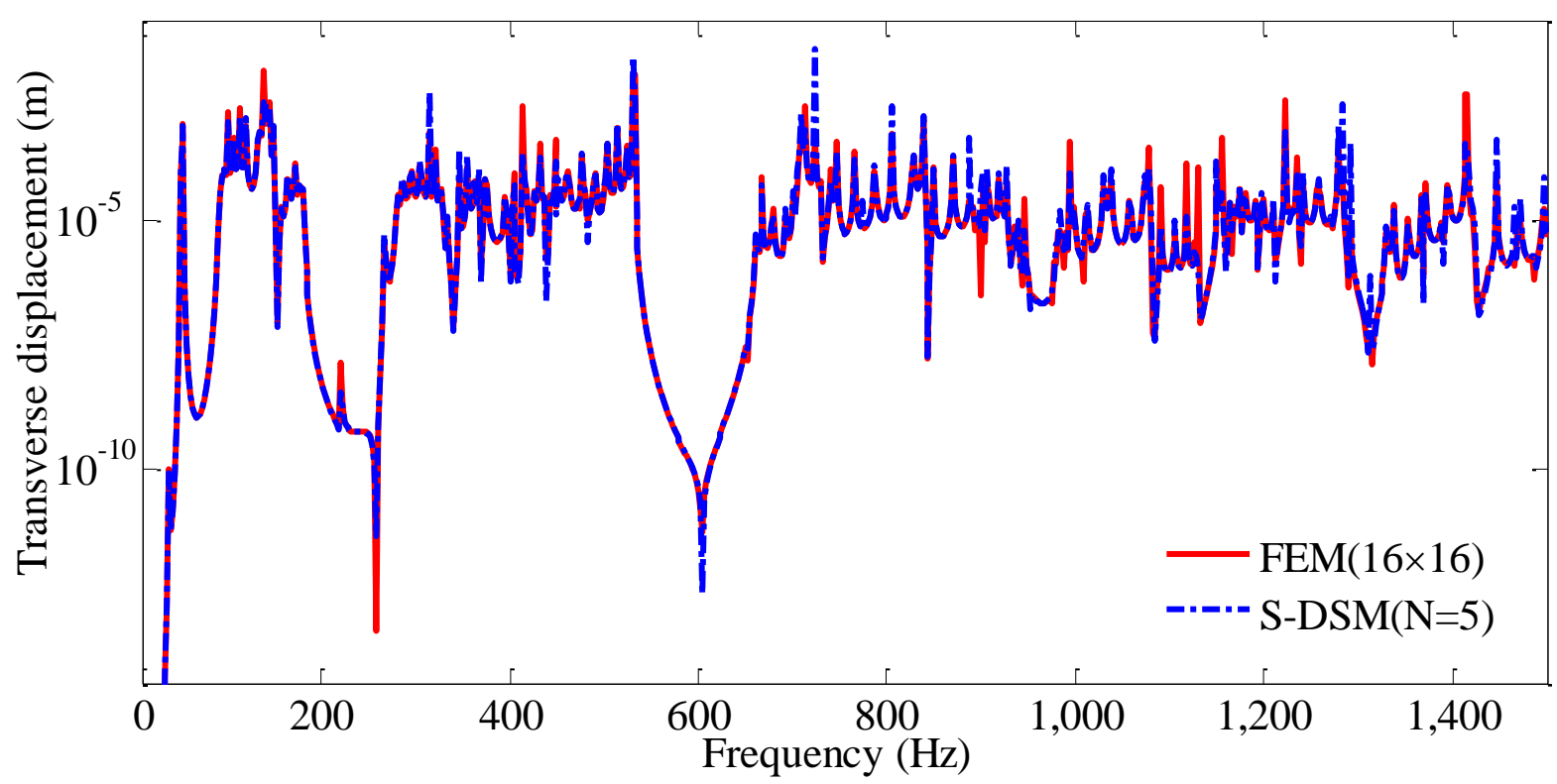

Fig. 12 Comparison of dynamic responses of the periodic plate structure with CF by the S-DSM and FEM. 


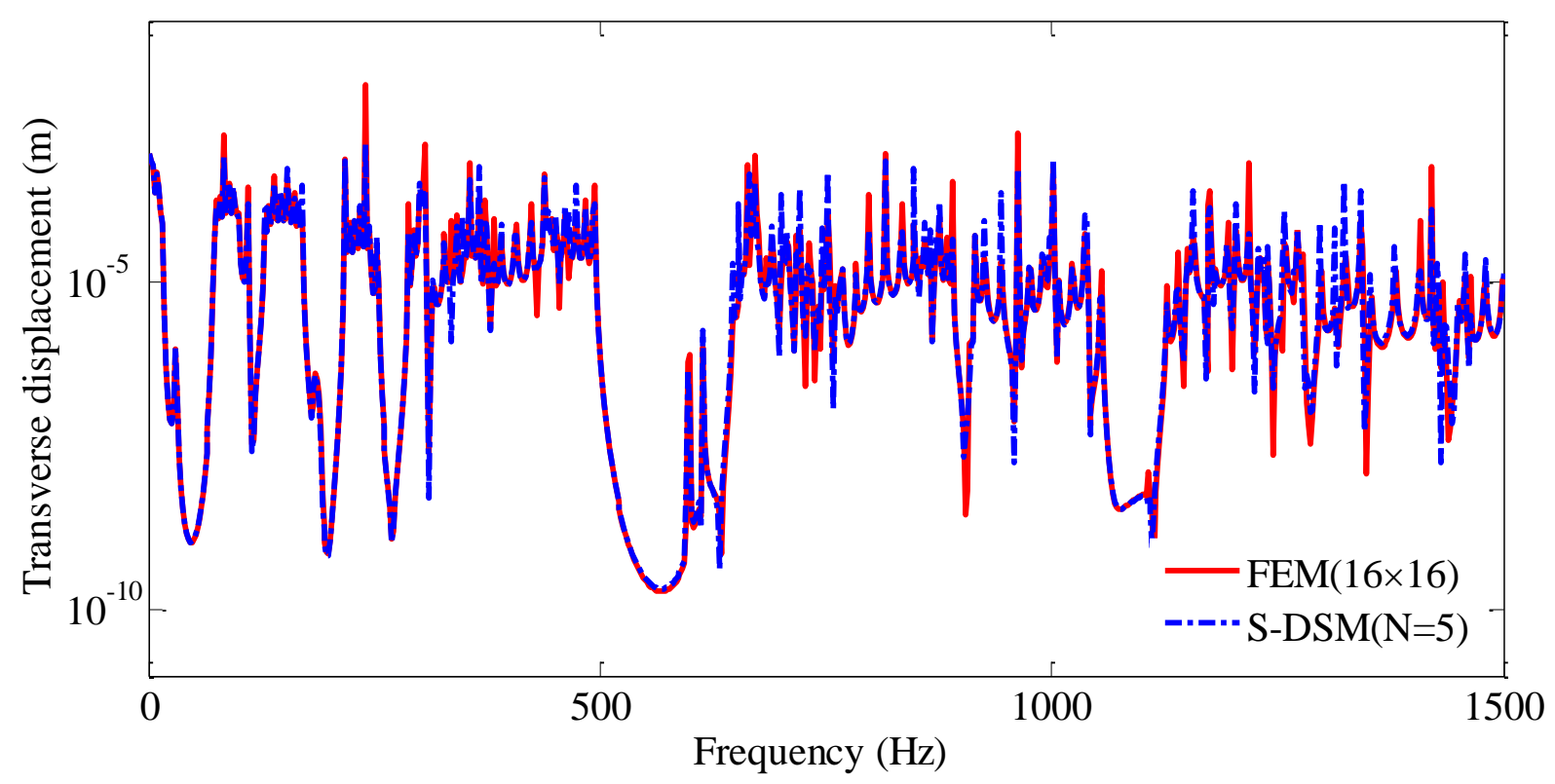

Fig. 13 Comparison of dynamic responses of the periodic plate structure with SF by the S-DSM and FEM.

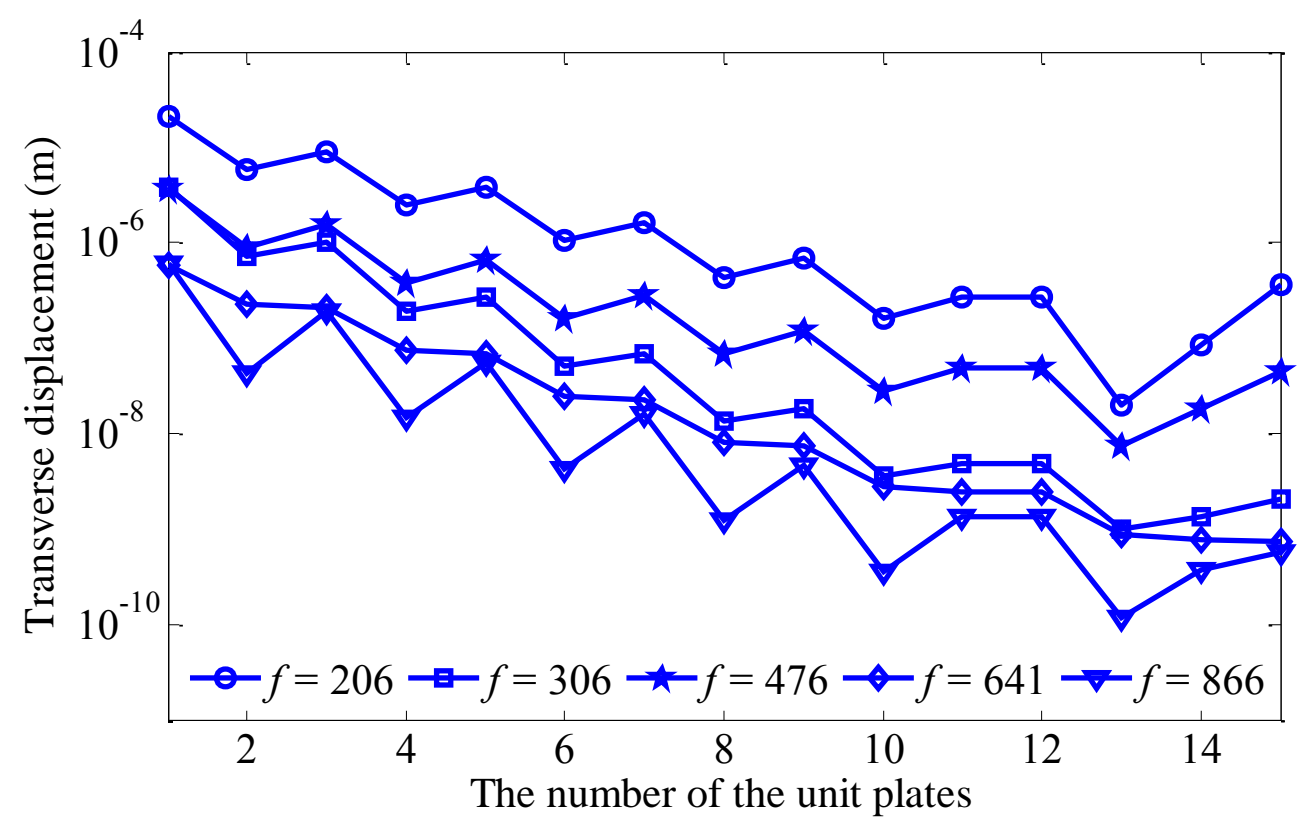

Fig. 14 Vibration responses for each unit plate for frequency located at the stop band. 


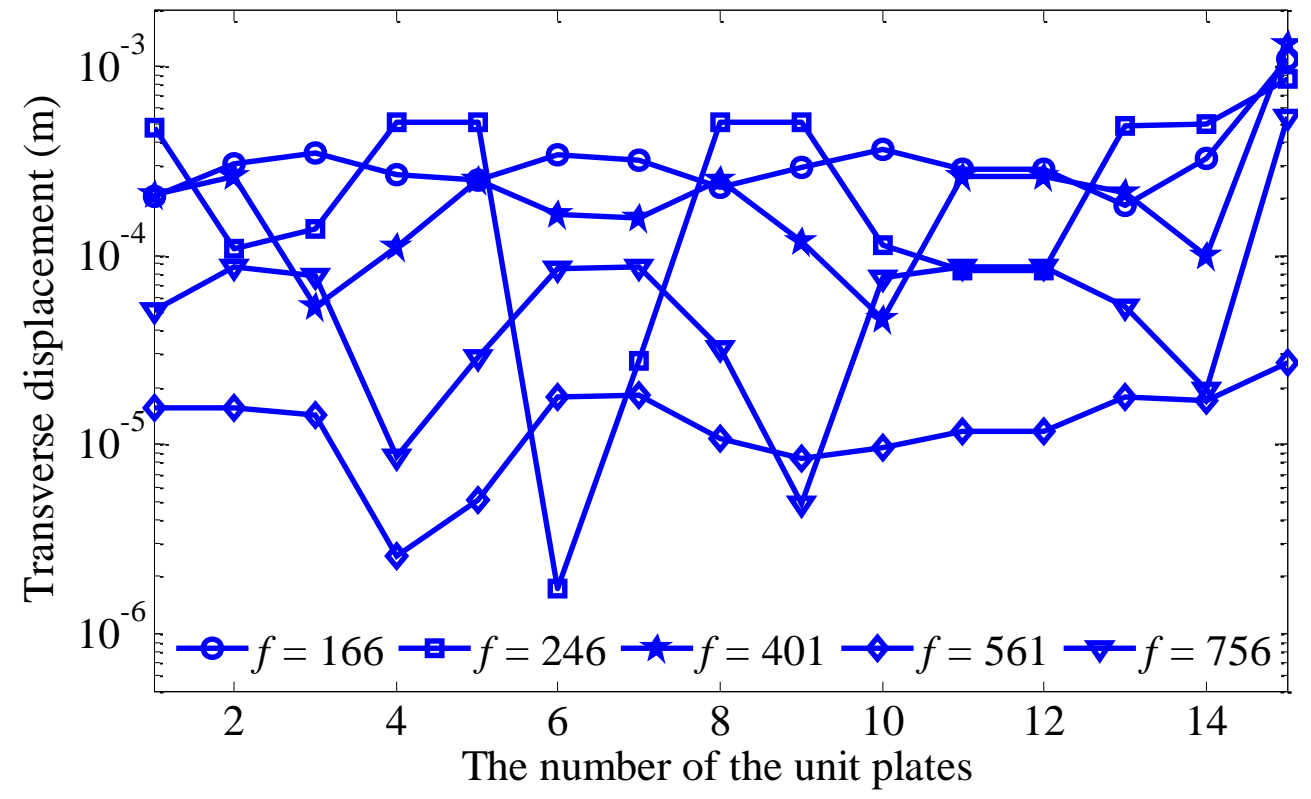

Fig. 15Vibration responses for each unit plate for frequency located at the pass band.

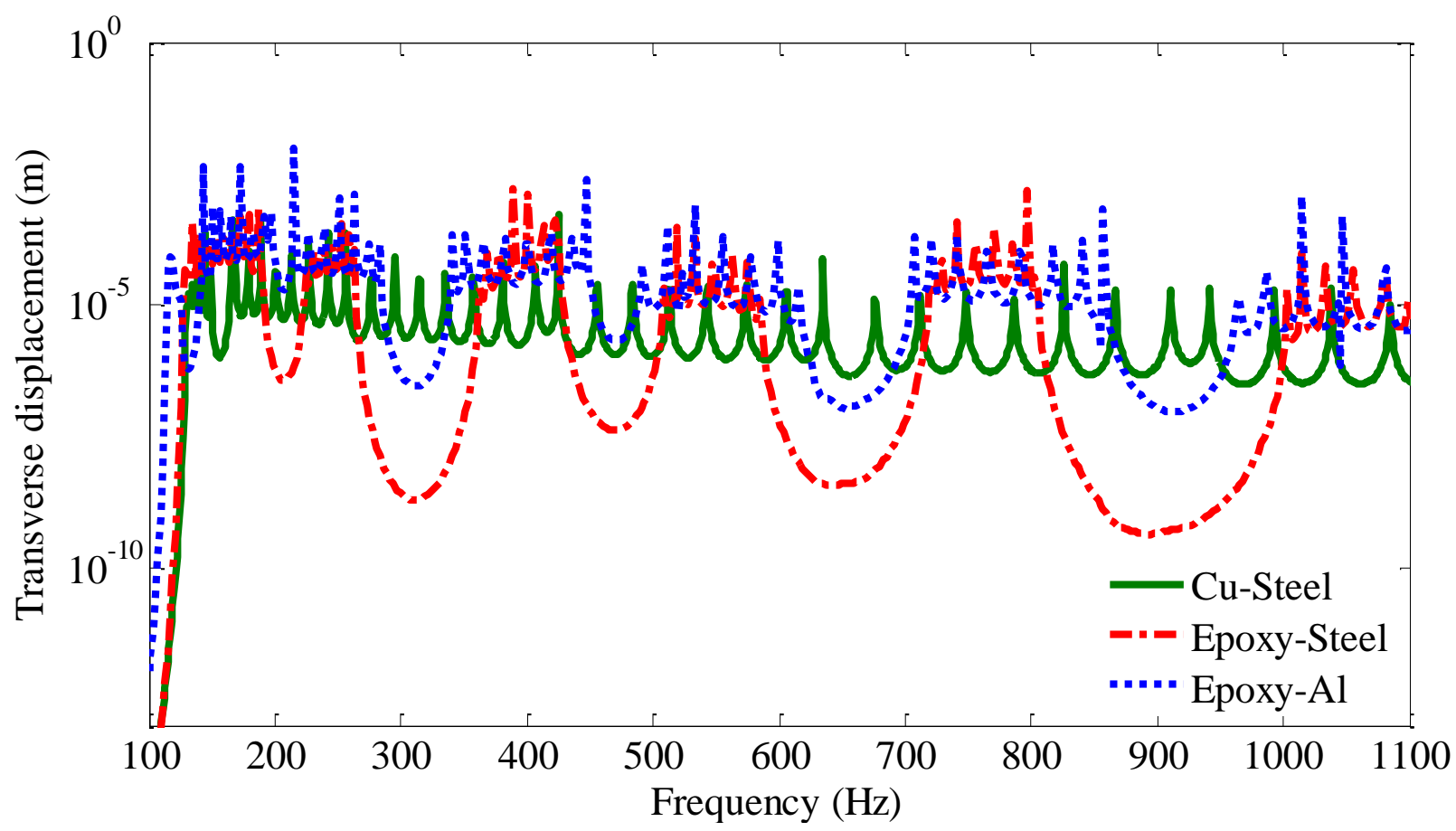

Fig.16 Comparison of frequency responses for periodic structure with different materials under CC boundary condition (Band gap rages: $150-160 \mathrm{~Hz}$; - - $191-224 \mathrm{~Hz}, 265-361 \mathrm{~Hz}, 429-507 \mathrm{~Hz}$, 807-1001Hz; $\cdots \cdot 285-339 H z, 451-489 H z, 619-703 \mathrm{~Hz}, 865-959 H z)$. 


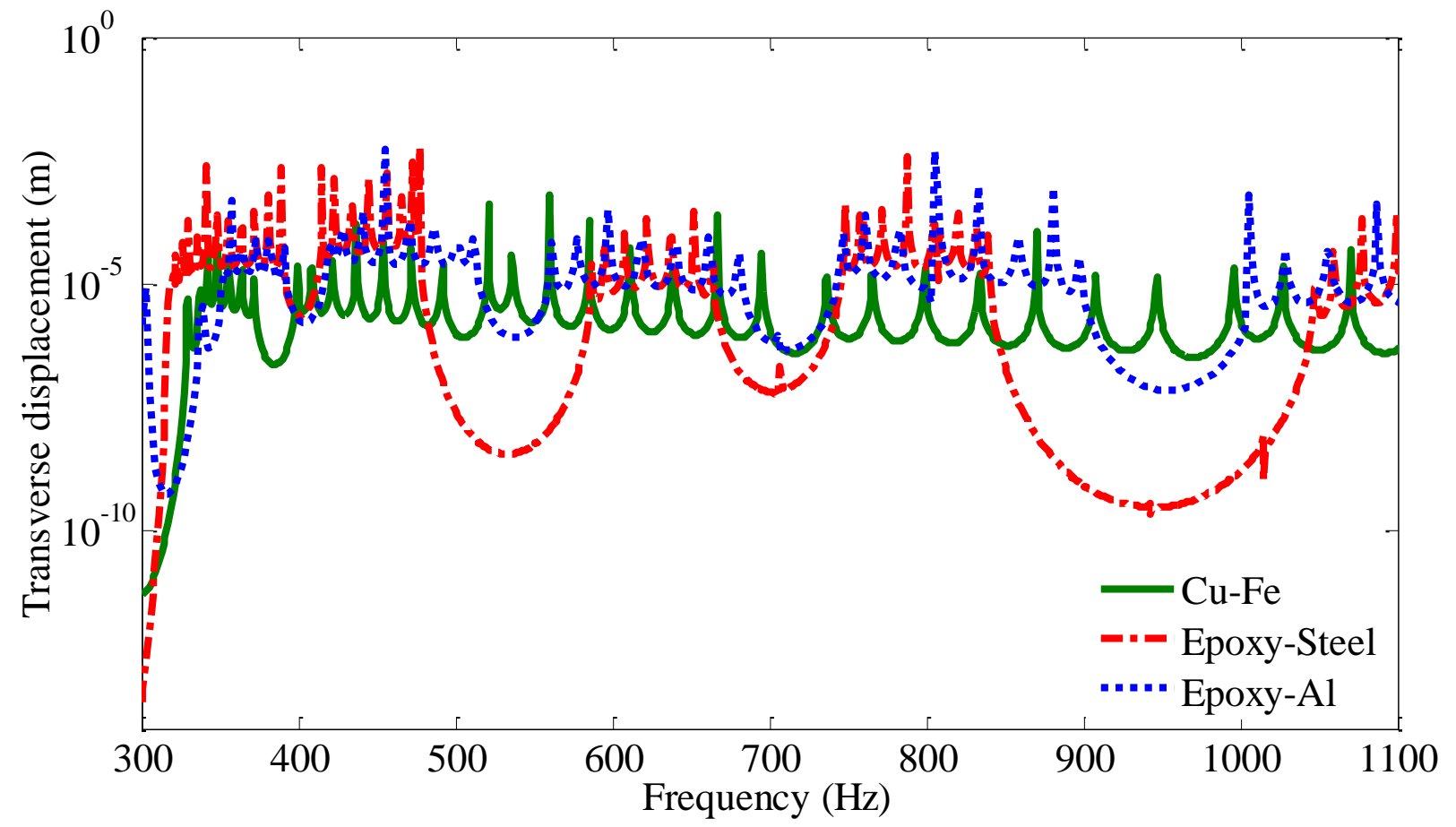

Fig.17 Comparison of frequency responses for periodic structure with different materials under SS boundary condition (Band gap rages: $-374-397 \mathrm{~Hz} ;=-391-413 \mathrm{~Hz}, 479-585 \mathrm{~Hz}, 666-744 \mathrm{~Hz}$, $841-1045 \mathrm{~Hz} ; \cdots \cdot 303-337 \mathrm{~Hz}, 393-417 \mathrm{~Hz}, 685-740 \mathrm{~Hz}, 901-1003 \mathrm{~Hz})$.

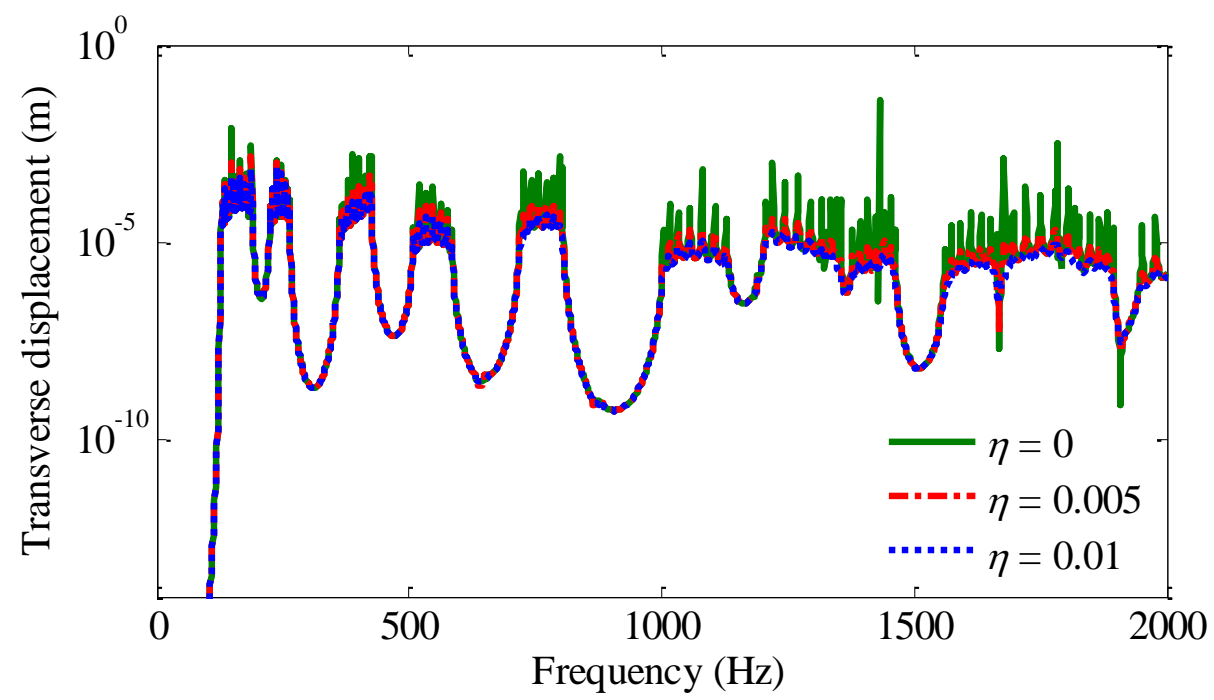

Fig .18 Damping effects on the vibration responses of the periodic plate structure. 


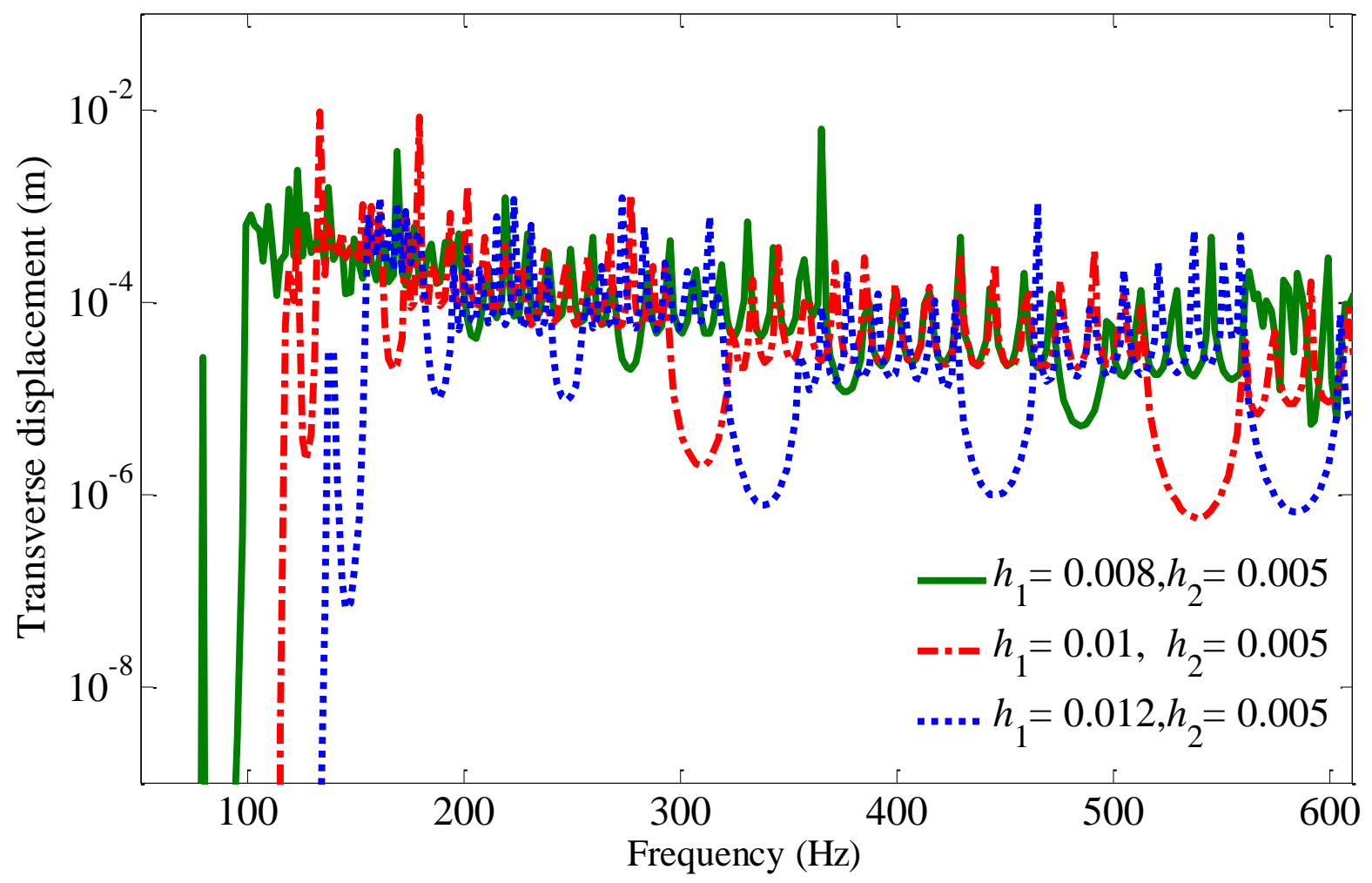

Fig. 19 Comparisons of band gaps for periodic plate structure with different thickness under CC boundary conditions (Band gap rages: $-270-280 \mathrm{~Hz}, 365-385 \mathrm{~Hz}, 475-495 \mathrm{~Hz}$; - $162-171 \mathrm{~Hz}, 295-323 \mathrm{~Hz}, 515-559 \mathrm{~Hz} ; \cdots \cdot \ldots 181-194 \mathrm{~Hz}, 240-254 \mathrm{~Hz}, 319-355 \mathrm{~Hz}, 429-463 \mathrm{~Hz}$, 561-602Hz). 


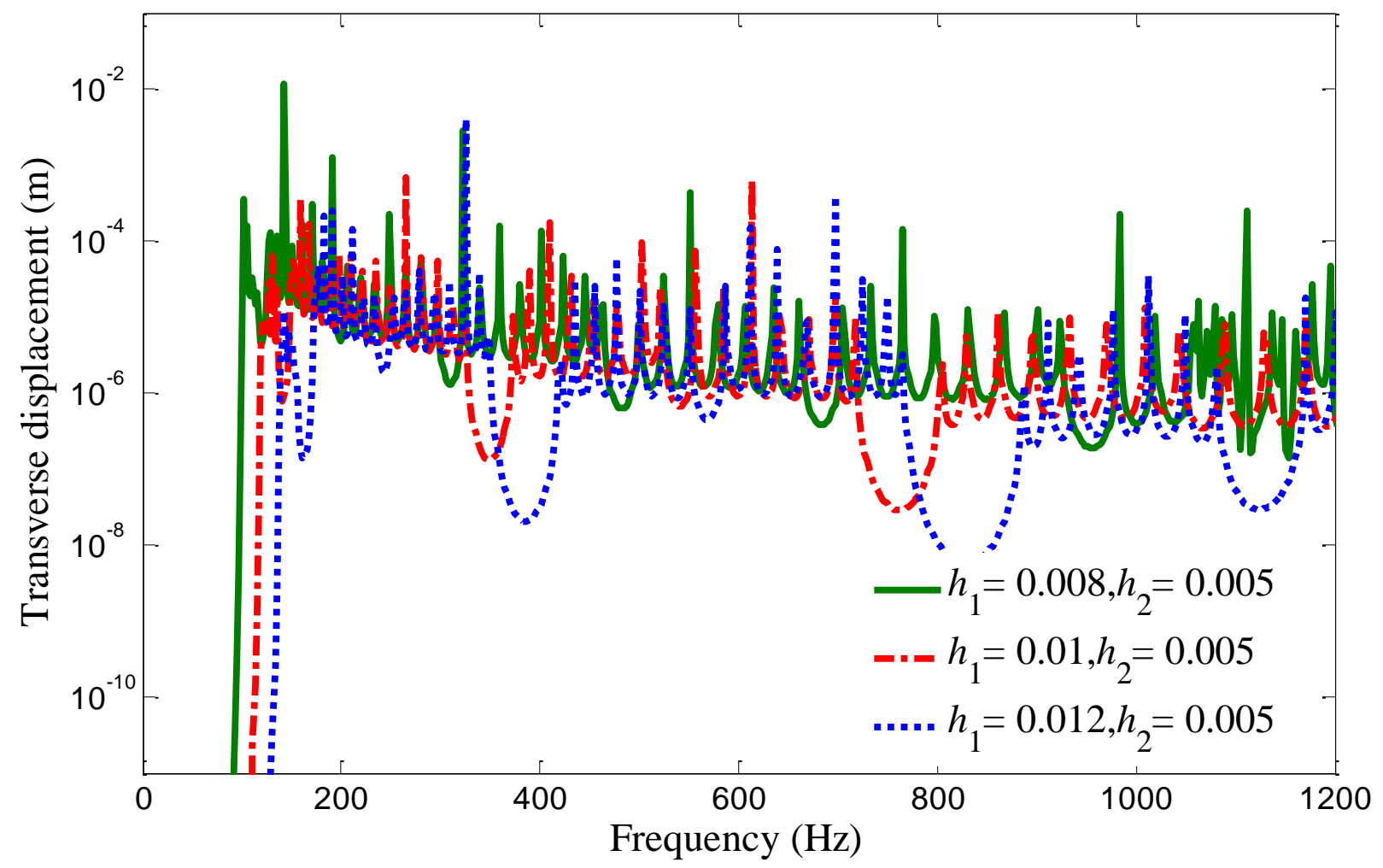

Fig. 20 Comparisons of band gaps for periodic plate structure with different thickness under SS boundary conditions (Band gap rages: - none; $\boldsymbol{-}=\mathbf{-} 327-371 \mathrm{~Hz}, 721-801 \mathrm{~Hz}$;

$351-419 \mathrm{~Hz}, 767-883 \mathrm{~Hz})$.

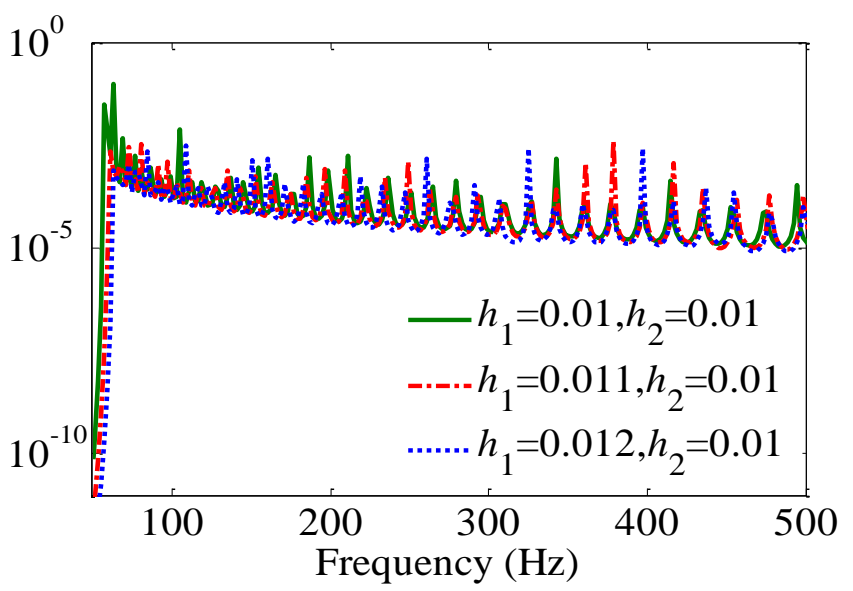

(a) Uniform material property.

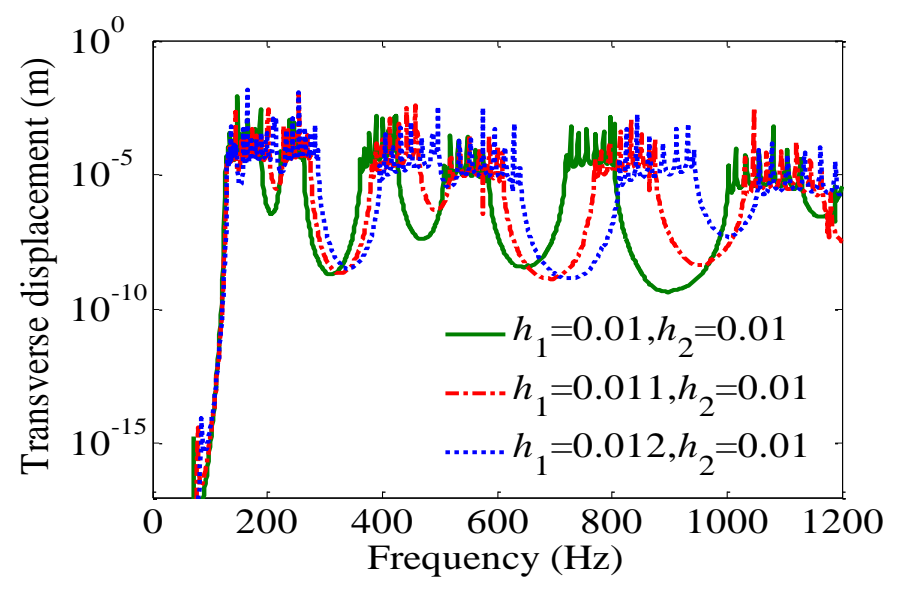

(b) Diferent material properties.

Fig. 21 The superposed influence of the geometrical and material properties on the band gaps. 


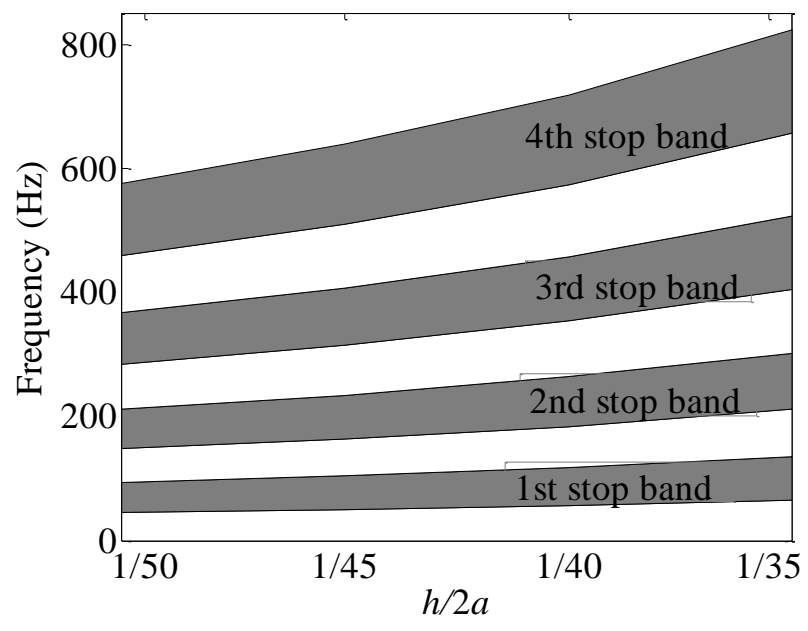

(a) CC boundary condition.

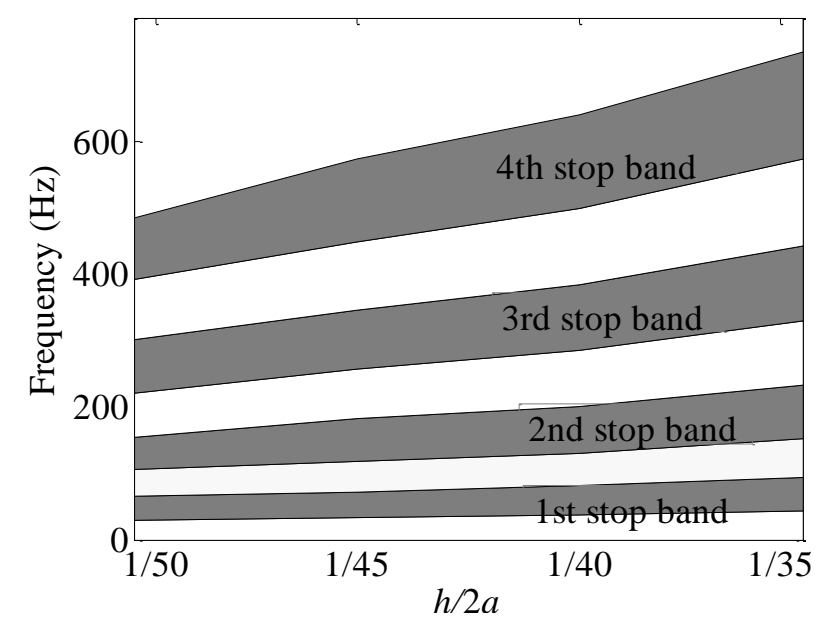

(b) SS boundary condition.

Fig. 22 The frequency ranges of the first four stop bands of the periodic plate.

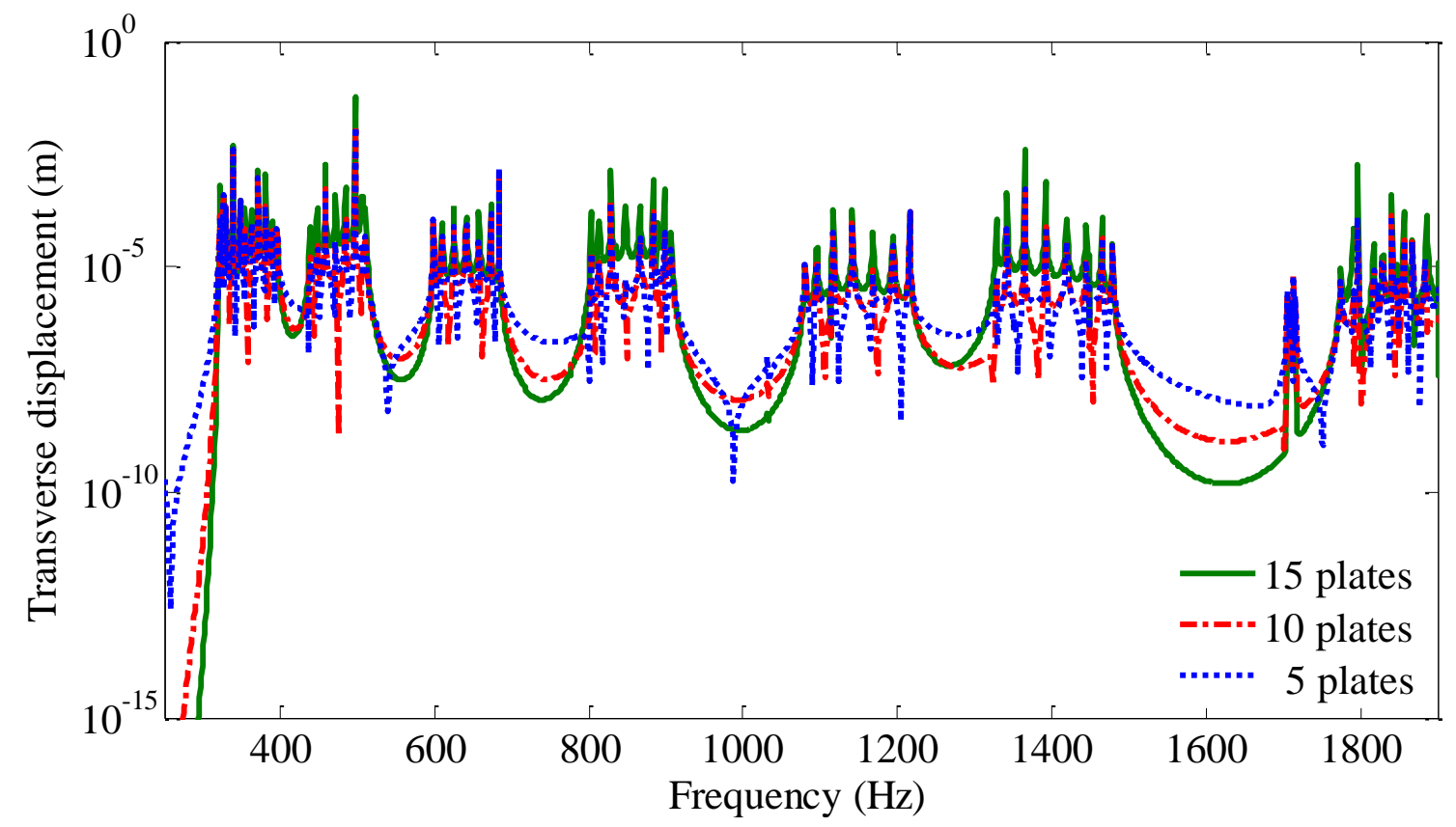

Fig. 23 Dynamic responses of periodic consisted of different number of unit cell under CC boundary condition. (Band gap rages: $389-438 \mathrm{~Hz}, 514-579 \mathrm{~Hz}, 686-800 \mathrm{~Hz}, 910-1078 \mathrm{~Hz}$, 


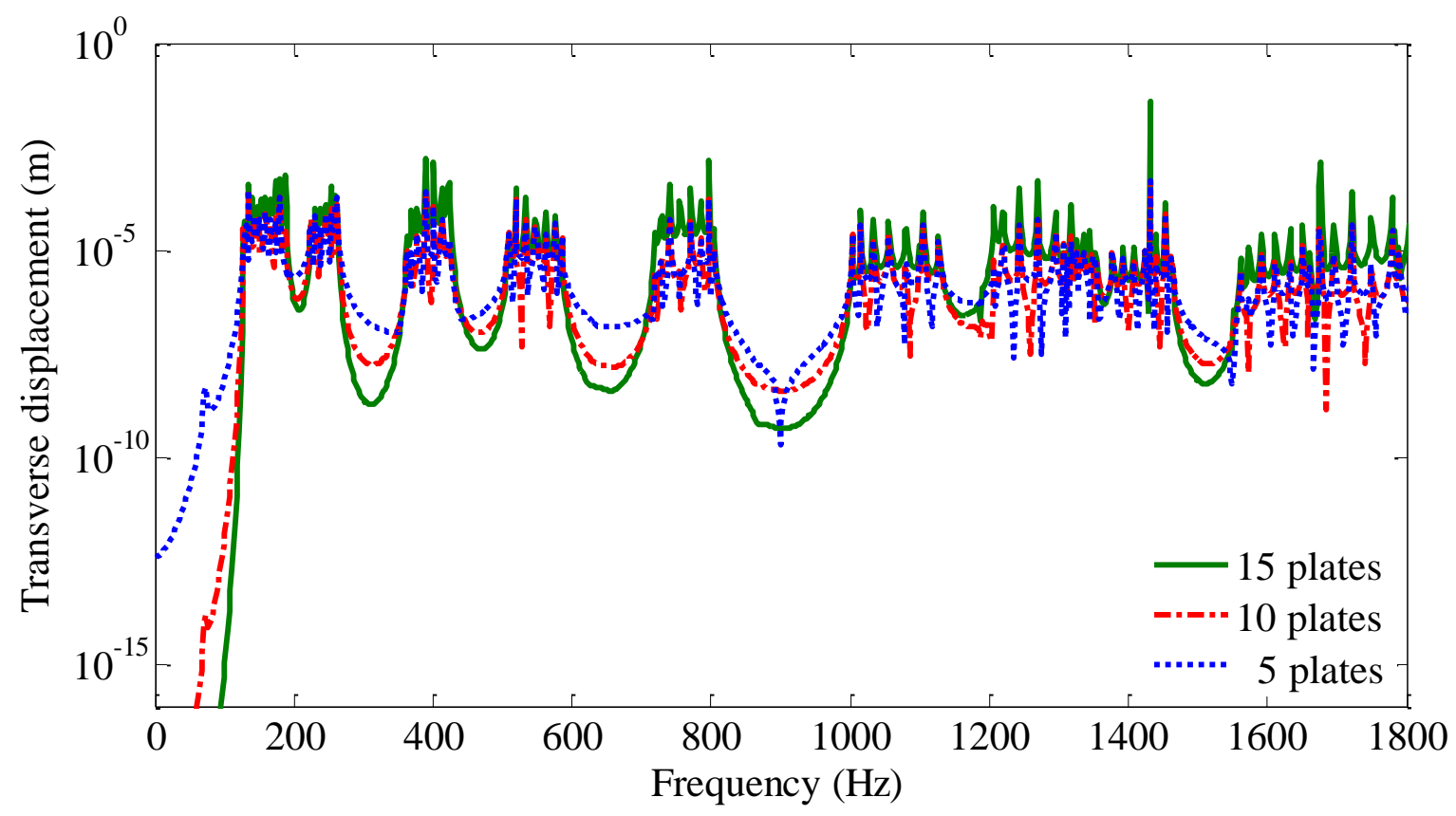

Fig. 24 Dynamic responses of periodic consisted of different number of unit cell under SS boundary condition(Band gap rages: 191-223Hz, 265-361Hz, 429-507Hz, 587-715Hz, 809-999Hz, 1133-1200Hz, 1465-1557Hz).

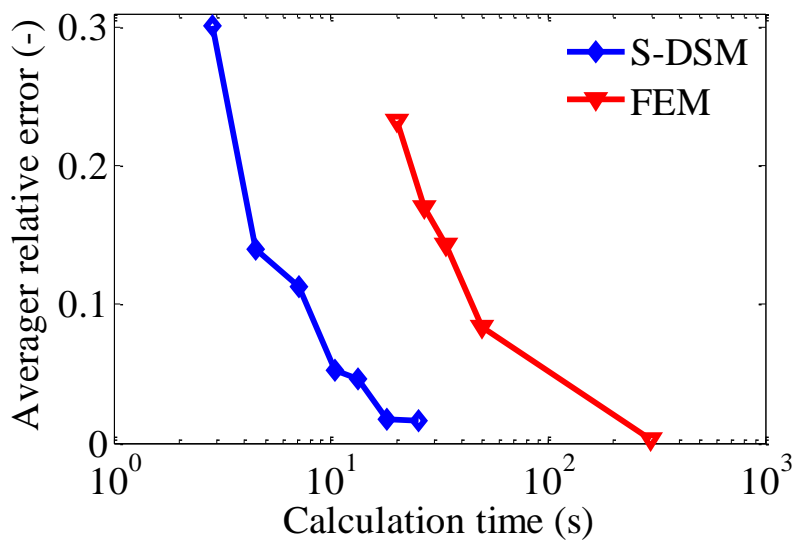

(a) S-DSM as reference

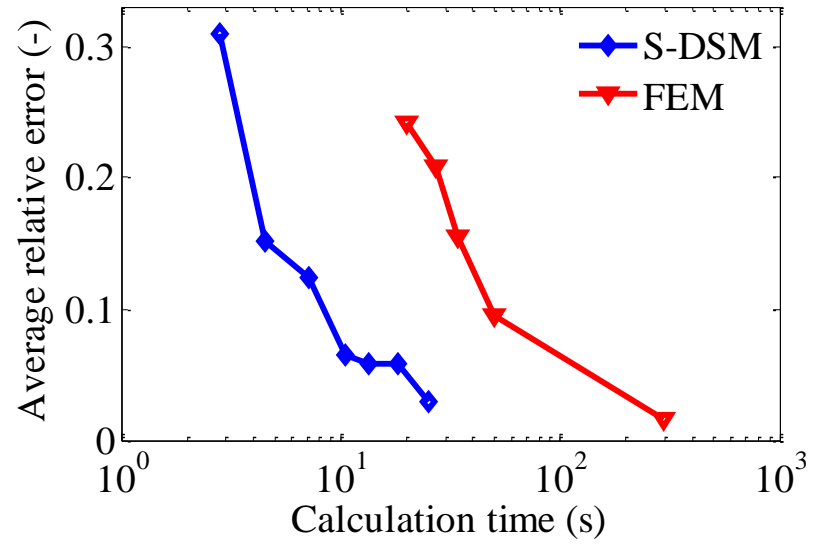

(b) FEM as reference

Fig. 25 Relative error as function of the calculation time. 
Table:

Table 1. Physical parameters of the materials.

\begin{tabular}{lcc}
\hline Materials & $\begin{array}{c}\text { Young's modulus } E \\
(\mathrm{GPa})\end{array}$ & $\begin{array}{c}\text { Density } \rho \\
\left(\mathrm{Kg} / \mathrm{m}^{3}\right)\end{array}$ \\
\hline Epoxy (Ep) & $4.35 \times 10^{9}$ & 1180 \\
Steel (Fe) & $200 \times 10^{9}$ & 7800 \\
Copper (Cu) & $127 \times 10^{9}$ & 8960 \\
Aluminum (Al) & $77.6 \times 10^{9}$ & 2730 \\
\hline
\end{tabular}

